\title{
One-pot, Two-step Synthesis of Substituted Anthraquinones from Chromium(0) Alkynyl Carbenes and Isobenzofurans
}

\author{
José Barluenga,* Silvia Martínez, Angel L. Suárez-Sobrino, and Miguel Tomás
}

Supporting Information:

\section{General}

All reactions involving air sensitive compounds were carried out under a $\mathrm{N}_{2}$ atmosphere (99.99\%). All glassware was oven-dried $\left(120^{\circ} \mathrm{C}\right)$, evacuated and purged with nitrogen. All common reagents and solvents were obtained from commercial suppliers and used without any further purification unless otherwise indicated. While 1,2-dihydro-1,4-epoxinaphtalene 1a was purchased, 1b was prepared following literature procedures. ${ }^{1}$ Alkenyl Fischer carbene $\mathbf{3}$ were prepared following described procedures. $^{2}$ Solvents were dried by standard methods and distilled prior to use. Flash column chromatography was carried out on silica gel 60, 230-240 mesh. NMR experiments were recorded on Bruker AC-200, AC-300 or DPX-300 spectrometers. Unless otherwise noted, ${ }^{1} \mathrm{H}$ NMR spectra were recorded in $\mathrm{CDCl}_{3}$ at $300.08 \mathrm{MHz}$ at $20^{\circ} \mathrm{C}$ with tetramethylsilane $(\delta=0.0)$ as the internal standard. ${ }^{13} \mathrm{C}$ NMR spectra were recorded in $\mathrm{CDCl}_{3}$ at $75.46 \mathrm{MHz}$ at $20^{\circ} \mathrm{C}$. ${ }^{1} \mathrm{H}$ NMR splitting pattern abbreviations are: $\mathrm{s}$, singlet; d, doublet; $\mathrm{t}$, triplet; $\mathrm{m}$, multiplet. ${ }^{13} \mathrm{C}$ NMR multiplicities were determined by DEPT, abbreviations are: q, $\mathrm{CH}_{3}$; t, $\mathrm{CH}_{2}$; d, $\mathrm{CH}$; s, quaternary carbons. COSY, HMSQC, HMBC and NOESY experiments were carried out on a Bruker AMX-400 spectrometer. Standard pulse sequences were employed for the DEPT experiments. High resolution mass spectra (HRMS) were obtained with a Finnigan Mat95 Mass Spectrometer and electron impact techniques (70 eV) were employed. Elemental analyses were carried out with a Perkin-Elmer 240 B microanalyzer.

\section{[4+2] Cycloaddition of alkynyl Fischer carbenes 3 and isobenzofurans 2.}

1,4-Dihydro-1,4-epoxynaphthalenes 1a,b (0.5 mmol) and 3,6-di-2-pyridyl-1,2,4,5-tetrazine (118 mg, 0.5 $\mathrm{mmol}$ ) were stirred at rt in THF $(6 \mathrm{~mL})$ for $20 \mathrm{~min}$ to generate the corresponding isobenzofurans $2 \mathbf{a}, \mathbf{b}$. Then, Fischer carbene $\mathbf{3}(0.5 \mathrm{mmol})$ was added and the mixture was stirred at rt for $2 \mathrm{~h}$ (3a-d), $4 \mathrm{~h}$ (3g, i), $7 \mathrm{~h}(3 \mathbf{e}, \mathbf{f})$ or $24 \mathrm{~h}$ (3h) until complete disappearance of the starting material (according to TLC). Finally, silica-gel was added and the crude mixture was purified by flash chromatography on silica-gel (Hexane/EtOAc 5:1) to obtain pure carbenes $\mathbf{4 a - j}$.<smiles>COC(=O)C1=C(C2=CCCC2)C2OC(C1=C(OC)C(C)(F)F)c1ccccc12</smiles>

4a: Yield: $72 \% .{ }^{1} \mathrm{H}$ NMR: $\delta 1.91$ (m, 2H), 2.48 (m, 4H), 4.33 (s, 3H), 5.86 (s, 1H), 5.98 (m, 1H), 6.09 (s, 1H), $7.13(\mathrm{~m}, 2 \mathrm{H}), 7.37(\mathrm{~m}, 2 \mathrm{H}) .{ }^{13} \mathrm{C}$ NMR: $\delta 23.7(\mathrm{t}), 33.4(\mathrm{t}), 33.7(\mathrm{t}), 65.7(\mathrm{q}), 85.7(\mathrm{~d}), 86.0(\mathrm{~d}), 120.0$ (d), 121.2 (d), 125.8 (d), 126.4 (s), 135.2 (d), 136.0 (s), 139.3 (s), 146.9 (s), 147.2 (s), 215.9 (s), 223.9 (s), 345.24 (s). Anal. Calcd for $\mathrm{C}_{22} \mathrm{H}_{16} \mathrm{CrO}_{7}$ : C, 59.47; H, 3.63. Found: C, 59.16; H, 3.75. HRMS calculated for $\mathrm{C}_{22} \mathrm{H}_{16} \mathrm{CrO}_{7}$ : 444.0301. Found: 444.0309 .<smiles>COC(=C(Cl)C(=O)[O-])C1=C(c2ccccc2)C2OC1c1ccccc12</smiles>

4d: Yield: 85\%. ${ }^{1} \mathrm{H}$ NMR: $\delta 4.26$ (s, 3H), 5.91 (s, 1H), 6.12 (s, 1H), 7.08-7.25 (m, 4H), 7.34-7.50 (m, 4H), $7.60(\mathrm{~m}, 1 \mathrm{H}) .{ }^{13} \mathrm{C}$ NMR: $\delta 65.9(\mathrm{q}), 85.9$ (d), 86.3 (d), 120.2 (d), 121.5 (s), 126.1 (d), 126.2 (d), 126.8 (d), 128.3 (s), 129.0 (d), 129.1 (d), 131.7 (d), 143.0 (s), 146.6 (s), 147.0 (s), 215.6 (s), 224.1 (s), 
346.7 (s). Anal. Calcd for $\mathrm{C}_{23} \mathrm{H}_{14} \mathrm{CrO}_{7}$ : C, 60.80; H, 3.11. Found: C, 60.61; H, 3.17. HRMS calculated for $\mathrm{C}_{23} \mathrm{H}_{14} \mathrm{CrO}_{7}$ : 454.0145. Found: 454.0139 .<smiles>COC(=O)C1=C(c2ccc(OC)cc2)C(=C(OC)C(F)(F)Cl)C2OC1c1ccccc12</smiles>

4e: Yield: $75 \% .{ }^{1} \mathrm{H}$ NMR $\left(\mathrm{CD}_{2} \mathrm{Cl}_{2}\right)$ : $\delta 3.78$ (s, 3H), 4.27 (s, 3H), 5.99 (s, 1H), 6.15 (s, 1H), 6.92 (m, $\left.2 \mathrm{H}\right)$, $7.10(\mathrm{~m}, 4 \mathrm{H}), 7.46(\mathrm{~m}, 1 \mathrm{H}), 7.65(\mathrm{~m}, 1 \mathrm{H}) .{ }^{13} \mathrm{C} \mathrm{NMR}\left(\mathrm{CD}_{2} \mathrm{Cl}_{2}\right): \delta 55.8(\mathrm{q}), 66.5(\mathrm{q}), 86.2(\mathrm{~d}), 86.8(\mathrm{~d})$, 114.1 (s), 115.0 (d), 120.4 (d), 121.6 (d), 124.6 (d), 126.2 (d), 128.9 (d), 130.7 (s), 143.9 (s), 147.5 (s), 154.8 (s), 161.0 (s), 216.3 (s), 224.8 (s), 347.3 (s). HRMS calculated for $\mathrm{C}_{24} \mathrm{H}_{16} \mathrm{CrO}_{8}$ : 484.0250. Found: 484.0259 .<smiles>COC(C1=C(c2cccc3ccccc23)C2OC1c1ccccc12)C(C)(F)F</smiles>

4f: Yield: 64\%. ${ }^{1} \mathrm{H}$ NMR: $\delta 4.29$ (s, 3H), 6.04 (s, 1H), 6.35 (s, 1H), 7.05-8.10 (m, 11H). ${ }^{13} \mathrm{C}$ NMR: $\delta 66.7$ (q), 86.8 (d), 88.7 (d), 121.5 (d), 121.9 (d), 125.7 (d), 126.3 (d), 126.4 (d), 126.7 (d), 126.8 (d), 127.2 (d), 129.1 (d), 129.9 (d), 131.2 (s), 131.5 (s), 134.1 (s), 147.4 (s), 147.8 (s), 150.1 (s), 162.6 (s), 216.1 (s), 224.2 (s), 341.4 (s). HRMS calculated for $\mathrm{C}_{27} \mathrm{H}_{16} \mathrm{CrO}_{7}$ : 504.0301. Found: 504.0296.<smiles>COC(=O)C(=C(Cl)C(=O)[O-])C1=C(OC)C2OC1c1c(OC)ccc(OC)c12</smiles>

4g: Yield: 81\%. ${ }^{1} \mathrm{H}$ NMR: $\delta 3.79$ (s, 3H), 3.83 (s, 3H), 4.00 (s, 3H), 6.64 (m, 2H), 7.24 (m, 2H), 7.30$7.43(\mathrm{~m}, 5 \mathrm{H}) .{ }^{13} \mathrm{C}$ NMR: $\delta 55.9(\mathrm{q}), 56.2(\mathrm{q}), 65.7$ (q), 83.7 (d), 84.5 (d), $111.6(\mathrm{~d}), 112.2(\mathrm{~d}), 127.1$ (d), 128.5 (s), 128.8 (d), 129.0 (d), 132.1 (s), 135.5 (s), 136.3 (s), 147.7 (s), 147.8 (s), 156.1 (s), 215.6 (s), 224.4 (s), 347.4 (s). HRMS calculated for $\mathrm{C}_{25} \mathrm{H}_{18} \mathrm{CrO}_{9}$ : 514.0356. Found: 514.0358.<smiles>COC(=C1C(c2ccc(Cl)cc2)=C(C(=O)OC(C)(F)F)C2OC1c1ccccc12)C(C)(F)F</smiles>

4h: Yield: 70\%. ${ }^{1} \mathrm{H}$ NMR: $\delta 4.34$ (s, 3H), 6.00 (s, 1H), 6.12 (s, 1H), 7.00-8.05 (m, 8H). ${ }^{13} \mathrm{C}$ NMR: $\delta 66.4$ (q), 86.3 (d), 86.9 (d), 120.5 (d), 121.1 (s), 122.0 (d), 126.6 (d), 128.3 (d), 129.0 (d), 129.8 (d), 130.3 (s), 132.0 (s), 135.5 (s), 146.8 (s), 156.9 (s), 215.9 (s), 224.5 (s), 346.8 (s). HRMS calculated for $\mathrm{C}_{23} \mathrm{H}_{13} \mathrm{ClCrO}_{7}$ : 487.9755. Found: 487.9746. 
<smiles>COC(=O)C1=C(C(C)(C)C)C(=C(OC)C(=O)O)C2OC1c1ccccc12</smiles>

4i: Yield: 60\%. ${ }^{1} \mathrm{H}$ NMR: $\delta 1.15$ (s, 9H), 4.45 (s, 3H), 5.79 (m, 2H), 7.18 (m, 2H), 7.39 (m, 1H), 7.74 (m, 1H). ${ }^{13}$ C NMR: $\delta 29.2$ (q), 34.2 (s), 66.2 (q), 85.9 (d), 86.4 (d), 120.9 (d), 121.8 (d), 126.0 (d), 126.3 (d), 147.7 (s), 148.1 (s), 151.0 (s), 152.7 (s), 216.5 (s), 222.1 (s), 351.1 (s). HRMS calculated for $\mathrm{C}_{21} \mathrm{H}_{18} \mathrm{CrO}_{7}$ : 434.0458. Found: 434.0451.<smiles>COC(=O)C(C)=C1C(=C(OC)c2ccccc2)C2CC1c1ccccc12</smiles>

4j: Yield: 74\%. ${ }^{1} \mathrm{H}$ NMR: $\delta 4.41$ (s, 3H), 6.00 (s, 1H), 6.13 (s, 1H), 7.10-7.21 (m, 4H), 7.41-7.59 (m, 4H), 7.61 (m, 1H). ${ }^{13} \mathrm{C}$ NMR: $\delta 67.7$ (q), 85.9 (d), 86.3 (d), 120.4 (d), 121.3 (d), 126.0 (d), 126.1 (d), 127.7 (d), 129.0 (d), 129.1 (d), 132.0 (s), 147.0 (s), 147.1 (s), 160.7 (s), 160.8 (s), 196.6 (s), 203.7 (s), 317.4 (s). HRMS calculated for $\mathrm{C}_{23} \mathrm{H}_{14} \mathrm{O}_{7} \mathrm{~W}$ : 586.0249. Found: 586.0235.

\section{Synthesis of aromatic systems 5a-g.}

Epoxynaphthalenes 1a,b $(0.5 \mathrm{mmol})$ and 3,6-di-2-pyridyl-1,2,4,5-tetrazine (118 $\mathrm{mg}, 0.5 \mathrm{mmol})$ were stirred at $\mathrm{rt}$ in THF $(6 \mathrm{~mL})$ for $20 \mathrm{~min}$. Then, Fischer carbenes $3(0.5 \mathrm{mmol})$ were added and the mixture was stirred at rt until complete disappearance of the starting material ( $2 \mathrm{~h}$, according to TLC). Then, tertbutylisocyanide $(114 \mu \mathrm{L}, 1.0 \mathrm{mmol})$ was added at $\mathrm{rt}$, and the mixture stirred for $1 \mathrm{~min}$, and heated at $60^{\circ} \mathrm{C}$ (for complexes 3c-e) or stirred at rt (for complexes 3a,b,f) for 12h. Finally, the solvent was removed and the products 5a-g were purified by flash chromatography on silica gel (hexanes/EtOAc, 10:1).<smiles>COc1c(NC(C)(C)C)c2c(c3c1C1OC3c3ccccc31)CCC2</smiles>

5a: Yield: 90\%. ${ }^{1} \mathrm{H}$ NMR: $\delta 1.18$ (s, 9H), 1.98-2.10 (m, 3H), 2.78-2.93 (m, 4H), 3.94 (s, 3H), $6.00(\mathrm{~s}, 1 \mathrm{H})$, $6.39(\mathrm{~s}, 1 \mathrm{H}), 7.02(\mathrm{~m}, 2 \mathrm{H}), 7.35(\mathrm{~m}, 2 \mathrm{H}) .{ }^{13} \mathrm{C}$ NMR: $\delta 27.0(\mathrm{t}), 31.0(\mathrm{q}), 31.1(\mathrm{t}), 33.3(\mathrm{t}), 55.6(\mathrm{~s}), 60.4(\mathrm{q})$, 81.7 (d), 82.1 (d), 120.3 (d), 120.5 (d), 126.1 (d), 131.8 (s), 132.9 (s), 133.4 (s), 138.5 (s), 140.1 (s), 148.2 (s), 148.5 (s), 148.7 (s). Anal. Calcd for $\mathrm{C}_{22} \mathrm{H}_{25} \mathrm{NO}_{2}$ : C, 78.77; H, 7.51; N 4.18. Found: C, 78.60; H, 7.49; $\mathrm{N}$, 4.23. HRMS calculated for $\mathrm{C}_{22} \mathrm{H}_{25} \mathrm{NO}_{2}: 335$.1885. Found: 335.1882 .<smiles>COc1c(NC(C)(C)C)c2c(c3c1C1OC3c3ccccc31)CCCC2</smiles>

5b: Yield: 35\%. ${ }^{1} \mathrm{H}$ NMR: $\delta$ 1,16 (s, 9H), 1.70-1.80 (m, 4H), 2.60 (m, 2H), 2.93 (m, 2H), 3.21 (bs, 1H), $3.92(\mathrm{~s}, 3 \mathrm{H}), 6.06(\mathrm{~s}, 1 \mathrm{H}), 6.40(\mathrm{~s}, 1 \mathrm{H}), 7.02(\mathrm{~m}, 2 \mathrm{H}), 7.31(\mathrm{~m}, 2 \mathrm{H}) .{ }^{13} \mathrm{C}$ NMR: $\delta 22.4(\mathrm{t}), 23.0(\mathrm{t}), 26.2(\mathrm{t})$, 27.4 (t), 30.8 (q), 54.9 (s), 59.5 (q), 80.8 (d), 81.9 (d), 119.9 (d), 124.8 (s), 125.7 (d), 131.53 (s), 133.0 (s), 
134.3 (s), 141.7 (s), 147.4 (s), 148.1 (s), 148.5 (s). HRMS calculated for $\mathrm{C}_{23} \mathrm{H}_{27} \mathrm{NO}_{2}$ : 349.2042. Found: 349.2044 .<smiles>COc1c(NC(C)(C)C)c(-c2ccccc2)cc2c1C1OC2c2ccccc21</smiles>

5c: Yield: 71\%. ${ }^{1} \mathrm{H}$ NMR: $\delta 0.87$ (s, 9H), 3.38 (bs, 1H), 4.03 (s, 3H), 6.01 (s, 1H), 6.48 (s, 1H), 6.94 (s, 1H), 7.05 (m, 2H), 7.30-7.40 (m, 7H). ${ }^{13} \mathrm{C}$ NMR: $\delta 30.3$ (q), 54.9 (s), 59.5 (q), 81.9 (d), 82.4 (d), 117.1 (d), 120.0 (d), 120.4 (d), 125.9 (d), 126.0 (d), 126.7 (d), 128.1 (d), 129.8 (d), 133.4 (s), 134.2 (s), 136.4 (s), 141.8 (s), 143.1 (s), 147.7 (s), 148.3 (s), 149.3 (s). HRMS calculated for $\mathrm{C}_{25} \mathrm{H}_{25} \mathrm{NO}_{2}$ : 371.1885. Found: 371.1879 .<smiles>COc1c2c(c3ccccc3c1NC(C)(C)C)C1OC2c2ccccc21</smiles>

5d: Yield: 88\%. ${ }^{1} \mathrm{H}$ NMR: $\delta 1.18$ (s, 9H), 3.41 (bs, 1H), 4.01 (s, 3H), 6.57 (m, 2H), 6.99 (m, 2H), 7.307.40 (m, 4H), 7.80 (m, 1H), 8.25 (m, 1H). ${ }^{13} \mathrm{C}$ NMR: $\delta 30.8$ (q), 56.0 (s), 60.5 (q), 81.4 (d), 82.6 (d), 120.1 (d), 120.3 (d), 122.5 (d), 124.5 (d), 124.6 (d), 125.6 (d), 125.7 (d), 126.4 (d), 131.0 (s), 132.1 (s), 137.9 (s), 143.1 (s), 147.4 (s), 147.5 (s), 148.5 (s), 148.7 (s). HRMS calculated for $\mathrm{C}_{23} \mathrm{H}_{23} \mathrm{NO}_{2}$ : 345.1729 . Found: 345.1723.<smiles>COc1ccc2c3c(c(OC)c(NC(C)(C)C)c2c1)C1OC3c2ccccc21</smiles>

5e: Yield: 75\%. ${ }^{1} \mathrm{H}$ NMR: $\delta 1.22$ (s, 9H), 3.92 (s, 3H), 4.03 (s, 3H), 6.50 (s, 1H), 6.56 (s, 1H), 6.98-7.06 (m, 3H), 7.36 (m, 2H), 7.69 (m, 2H). ${ }^{13} \mathrm{C}$ NMR: $\delta 30.7$ (q), 54.1 (s), $55.3(\mathrm{q}), 60.4(\mathrm{q}), 81.4(\mathrm{~d}), 82.7(\mathrm{~d})$, 104.5 (d), 110.0 (s), 113.8 (s), 118.1 (d), 119.9 (d), 120.3 (d), 124.0 (d), 125.8 (d), 130.2 (s), 133.5 (s), 134.4 (s), 148.2 (s), 148.3 (s), 148.8 (s), 157.0 (s). HRMS calculated for $\mathrm{C}_{24} \mathrm{H}_{25} \mathrm{NO}_{3}$ : 375.1834. Found: 375.1841 .<smiles>COc1c2c(c3c(ccc4ccccc43)c1NC(C)(C)C)C1OC2c2ccccc21</smiles>

5f: Yield: 85\%. ${ }^{1} \mathrm{H}$ NMR: $\delta 1.21$ (s, 9H), 3.62 (bs, 1H), 4.08 (s, 3H), 6.57 (s, 1H), 7.07 (s, 1H), 7.12 (m, 2H), 7.41-7.90 (m, 6H), 8.33 (d, $J=9.46 \mathrm{~Hz}, 1 \mathrm{H}), 8.53$ (d, $J=7.63 \mathrm{~Hz}, 1 \mathrm{H}) .{ }^{13} \mathrm{C}$ NMR: $\delta 31.2(\mathrm{q}), 56.4$ (s), 61.4 (q), 81.4 (d), 84.0 (d), 120.9 (d), 121.0 (d), 123.2 (s), 125.2 (d), 125.9 (d), 126.0 (d), 126.3 (d), 126.5 (d), 126.6 (d), 126.7 (d), 128.8 (d), 130.3 (s), 130.7 (s), 132.6 (s), 132.9 (s), 137.4 (s), 142.5 (s), 148.1 (s), 148.6 (s), 149.2 (s). HRMS calculated for $\mathrm{C}_{27} \mathrm{H}_{25} \mathrm{NO}_{2}$ : 395.1885. Found: 395.1874. 
<smiles>COc1ccc(OC)c2c1C1OC2c2c1c(NC(C)(C)C)c1ccccc1c2OC</smiles>

5g: Yield: 85\%. ${ }^{1} \mathrm{H}$ NMR: $\delta 1.19$ (s, 9H), 3.77 (s, 3H), 3,78 (s, 3H), 4.08 (s, 3H), 6.50 (m, 2H), 6.77 (m, 2H), 7.30 (m, 2H), 7.85 (m, 1H), 8.34 (m, 1H). ${ }^{13} \mathrm{C}$ NMR: $\delta 31.2(\mathrm{q}), 56.3(\mathrm{~s}), 56.4(\mathrm{q}), 56.7$ (q), 60.5 (q), 79.7 (d), 81.2 (d), 111.7 (d), 112.2 (d), 123.2 (d), 124.6 (d), 124.7 (d), 124.8 (s), 126.6 (d), 130.7 (s), 132.3 (s), 137.2 (s), 137.7 (s), 137.9 (s), 143.8 (s), 147.6 (s), 148.0 (s), 148.4 (s). Anal. Calcd for $\mathrm{C}_{25} \mathrm{H}_{27} \mathrm{NO}_{4}$ : C, 74.05; H, 6.71; N, 3.45. Found: C, 74.35; H, 6.58; N, 3.57. HRMS calculated for $\mathrm{C}_{25} \mathrm{H}_{27} \mathrm{NO}_{4}$ : 405.1940. Found: 405.1931.

\section{Photochemical cyclization of carbene complex 4a to dihydrotetraphene 6}

A solution of carbene complex 4d (200mg, $0.44 \mathrm{mmol}$ ) in THF (80 mL) was introduced in a sealed tube under carbon monoxide atmosphere and purged with this gas for $10 \mathrm{~min}$. Next, it was irradiated with a $400 \mathrm{~W}$ pressure mercury lamp for $1 \mathrm{~h}$ at rt, and then stirred under $\mathrm{CO}$ atmosphere for $12 \mathrm{~h}$. Solvent was removed and the crude mixture was purified by flash chromatography on silica-gel (Hexane/EtOAc 5:1) to give dihydrotetraphene $\mathbf{6}$ as a single product.<smiles>COc1c2c(c3ccccc3c1O)C1OC2c2ccccc21</smiles>

6: Yield: 84\%. ${ }^{1} \mathrm{H}$ NMR: $\delta 4.08$ (s, 3H), 6.13 (s, 1H), 6.53 (m, 2H), 6.99 (m, 2H), 7.39 (m, 4H), 7.81 (d, $J$ $=8.00 \mathrm{~Hz}, 1 \mathrm{H}), 8.06(\mathrm{~d}, J=8.00 \mathrm{~Hz}, 1 \mathrm{H}) .{ }^{13} \mathrm{C}$ NMR: $\delta 61.4(\mathrm{q}), 81.3$ (d), 82.2 (d), 120.0 (d), 120.1 (d), 122.4 (s), 122.5 (d), 122.7 (d), 124.3 (s), 124.8 (d), 125.5 (d), 125.6 (d), 125.8 (d), 137.1 (s), 137.8 (s), 137.9 (s), 140.8 (s), 148.4 (s), 148.7 (s). Anal. Calcd for $\mathrm{C}_{19} \mathrm{H}_{14} \mathrm{O}_{3}$ : C, 78.61; H, 4.86. Found: C, 78.72; H, 4.71. HRMS calculated for $\mathrm{C}_{19} \mathrm{H}_{14} \mathrm{O}_{3}$ : 290.0943. Found: 290.0949.

\section{Cyclopentannulation reaction of carbene $4 \mathrm{~h}$ to tetracyclic compound 7.}

Epoxynaphtalene 1a (72 mg, $0.5 \mathrm{mmol})$ and 3,6-di-2-pyridyl-1,2,4,5-tetrazine (118 mg, $0.5 \mathrm{mmol})$ were stirred at rt in THF $(6 \mathrm{~mL})$ for $20 \mathrm{~min}$. Then, Fischer enynylcarbene $3 \mathbf{b}(0.5 \mathrm{mmol})$ was added at $0^{\circ} \mathrm{C}$ and the mixture was at this temperature for $1 \mathrm{~h}$ until complete formation of carbene [4+2] cycloadduct (according to TLC), then it was allowed to reach rt and stirred for $4 \mathrm{~h}$. A $6 \mathrm{~N} \mathrm{HCl}$ solution (4 mL) was added and the crude mixture stirred for $30 \mathrm{~min}$. After ethyl ether extraction and solvent removal the crude product was purified by chromatography on silica-gel (Hexane/EtOAc 1:1) to give 7.<smiles>O=C1C2=C(CCCC2)c2cc3ccccc3cc21</smiles>

7: Yellow solid. Yield: 75\%. ${ }^{1} \mathrm{H}$ NMR: $\delta 1.78-1.87$ (m, 4H), 2.34 (m, 2H), 2.58 (m, 2H), 7.24 (s, $\left.1 \mathrm{H}\right)$, 7.50 (m, 2H), 7.73-7.80 (m, 2H), 7.82 (s, 1H). ${ }^{13} \mathrm{C}$ NMR: $\delta 20.1(\mathrm{t}), 21.9(\mathrm{t}), 23.0(\mathrm{t}), 117.1(\mathrm{~d}), 122.7$ (d), 126.6 (d), 128.3 (d), 128.8 (d), 130.7 (d), 131.3 (s), 133.5 (s), 136.2 (s), 138.5 (s), 140.1 (s), 159.0 (s), 192.0 (s). Anal. Calcd for $\mathrm{C}_{17} \mathrm{H}_{14} \mathrm{O}$ : C, 87.15; H, 6.02. Found: C, 87.31; H, 6.13. HRMS calculated for $\mathrm{C}_{17} \mathrm{H}_{14} \mathrm{O}$ : 234.1045. Found: 234.1037. 


\section{Synthesis of phenols 8a,b and the corresponding quinones 9a,b.}

Compounds 5a,d $(0.3 \mathrm{mmol})$ were dissolved in THF $(6 \mathrm{~mL})$ and a solution of $t$-BuLi $(0.75 \mathrm{~mL}, 0.75$ mmol, 1M/pentane) in hexane (6 mL) was added dropwise. After stirring for 20 min, saturated $\mathrm{NH}_{4} \mathrm{Cl}$ solution was poured into the mixture, followed by ethyl ether extraction to give phenols $\mathbf{8 a}, \mathbf{b}$, that showed to be unstable. However, 8b could be purified for full NMR characterization after a fast chromatography on silica-gel (Hexane/ $\mathrm{CH}_{2} \mathrm{Cl}_{2} 1: 2$ ).

The crude 8a,b were dissolved in $\mathrm{MeOH}(6 \mathrm{~mL})$, treated with $\mathrm{K}_{2} \mathrm{CO}_{3}(132 \mathrm{mg}, 0,96 \mathrm{mmol})$ and stirred overnight at rt under an oxygen atmosphere. After hydrolysis with saturated $\mathrm{NH}_{4} \mathrm{Cl}$ solution, extraction with EtOAc and solvent removal, quinones 9a,b were purified by flash chromatography on silica-gel (Hexane/ $\mathrm{CH}_{2} \mathrm{Cl}_{2}$ 1:2).<smiles>COc1c(NC(C)(C)C)c2ccccc2c2cc3ccccc3c(O)c12</smiles>

8b: (unstable). ${ }^{1} \mathrm{H}$ NMR: $\delta 1.36$ (s, 9H), 4.11 (s, 3H), 7.50-7.59 (m, 4H), 8.00-8.22 (m, 2H), 8.50 (m, 1H), $8.65(\mathrm{~s}, 1 \mathrm{H}), 8.80(\mathrm{~m}, 1 \mathrm{H}), 10.94(\mathrm{~s}, 1 \mathrm{H}) .{ }^{13} \mathrm{C}$ NMR: $\delta 30.8(\mathrm{q}), 55.8(\mathrm{~s}), 60.4(\mathrm{q}), 110.6(\mathrm{~s}), 112.2(\mathrm{~d})$, 122.0 (s), 122.3 (d), 123.4 (d), 123.6 (d), 124.6 (d), 126.0 (d), 126.4 (d), 127.0 (d), 127.8 (d), 128.3 (s), 129.1 (s), 129.4 (s), 132.3 (s), 132.9 (s), 148.4 (s). 150.0 (s).<smiles>COc1c(NC(C)(C)C)c2c(c3c1C(=O)c1ccccc1C3=O)CCC2</smiles>

9a: Yield: 71\%. ${ }^{1} \mathrm{H}$ NMR: $\delta 1.36$ (s, 9H), 2.13 (m, 2H), 2.95 (m, 2H), 3.49 (m, 2H), 3.88 (s, 3H), 4.37 (bs, 1H), $7.69(\mathrm{~m}, 2 \mathrm{H}), 8.21(\mathrm{~m}, 2 \mathrm{H}) .{ }^{13} \mathrm{C}$ NMR: $\delta 27.5(\mathrm{t}), 33.7(\mathrm{q}), 35.7(\mathrm{t}), 37.7(\mathrm{t}), 57.2(\mathrm{~s}), 63.3(\mathrm{q})$, 125.5 (s), 127.1 (s), 128.7 (d), 129.1 (d), 135.5 (d), 135.6 (d), 136.2 (s), 137.0 (s), 145.4 (s), 147.3 (s), 147.7 (s), 154.1 (s), 186.0 (s), 186.2 (s). Anal. Calcd for $\mathrm{C}_{22} \mathrm{H}_{23} \mathrm{NO}_{3}$ : C, 75.62; H, 6.63; N, 4.01. Found: C, 75.77; H, 6.65; N, 4.19. HRMS calculated for $\mathrm{C}_{22} \mathrm{H}_{23} \mathrm{NO}_{3}$ : 349.1678. Found: 349.1672.<smiles>COc1c2c(c3ccccc3c1NC(C)(C)C)C(=O)c1ccccc1C2=O</smiles>

9b: Yield: 69\%. ${ }^{1} \mathrm{H}$ NMR: $\delta 1.28$ (s, 9H), 3.93 (s, 3H), 4.40 (bs, 1H), 7.50-7.73 (m, 2H), 7.73 (m, 2H), 8.16-8.21 (m, 2H), 8.33 (d, $J=8.49 \mathrm{~Hz}, 1 \mathrm{H}), 9.51$ (d, $J=8.85 \mathrm{~Hz}, 1 \mathrm{H}) .{ }^{13} \mathrm{C}$ NMR: $\delta 31.3(\mathrm{q}), 57.0(\mathrm{~s})$, 61.9 (q), 125.7 (d), 126.2 (d), 126.5 (d), 127.1 (d), 128.2 (s), 128.3 (d), 128.8 (d), 129.2 (s), 133.1 (d), 133.6 (d), 133.7 (s), 134.4 (s), 134.6 (s), 139.8 (s), 145.0 (s), 148.5 (s), 184.7 (s), 185.6 (s). HRMS calculated for $\mathrm{C}_{23} \mathrm{H}_{21} \mathrm{NO}_{3}$ : 359.1521. Found: 359.1515 .

\section{Synthesis of phenol 8c}

A solution of compound $5 \mathrm{~g}$ (132 $\mathrm{mg}, 0.33 \mathrm{mmol})$ in THF $(6 \mathrm{~mL})$ was treated with 2,6-lutidine $(0.75 \mathrm{~mL}$, $6,6 \mathrm{mmol})$ in $\mathrm{CH}_{2} \mathrm{Cl}_{2}(15 \mathrm{~mL})$ and cooled at $-78^{\circ} \mathrm{C}$. Then, TBSOTf was added and the reaction mixture stirred at rt for $12 \mathrm{~h}$. The mixture was quenched with brine and extracted with $\mathrm{CH}_{2} \mathrm{Cl}_{2}$ and the crude purified by flash chromatography on silica-gel (Hexane/EtOAc 10:1) to give the silylated 8c in 91\% 
yield. Finally, it was deprotected by treatment with TBAF (86 mg, $0.33 \mathrm{mmol})$ in $\mathrm{THF}(6 \mathrm{~mL})$ at $0^{\circ} \mathrm{C}$ for $5 \mathrm{~min}$, followed by extraction with EtOAc, solvent removal and flash chromatography on silica-gel (Hexane/EtOAc 1:1) to give phenol 8c.

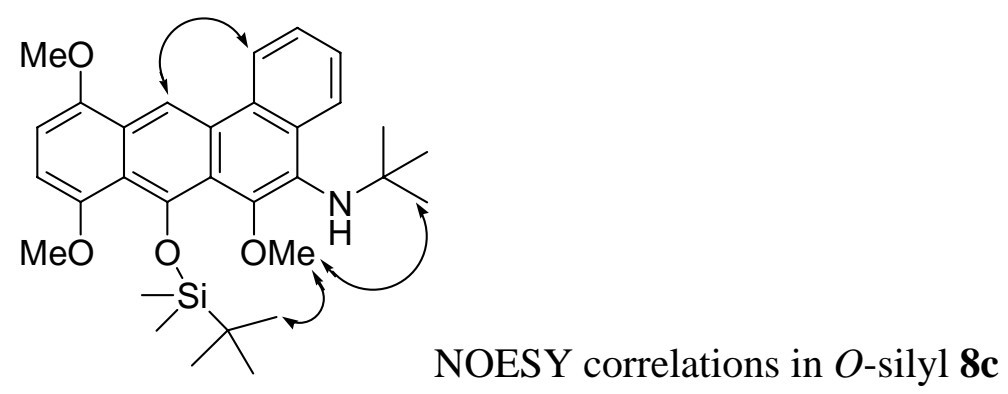

O-Silyl 8c: Yield: 91\%. ${ }^{1} \mathrm{H}$ NMR: $\delta-0.21$ (s, 6H), 1.16 (s, 9H), 1.38 (s, 9H), 3.85 (s, 3H), 3.92 (s, 3H), 4.07 (s, 3H), $6.68(\mathrm{~m}, 2 \mathrm{H}), 7.57(\mathrm{~m}, 2 \mathrm{H}), 8.38(\mathrm{~m}, 1 \mathrm{H}), 8.80(\mathrm{~m}, 1 \mathrm{H}), 9.24(\mathrm{~s}, 1 \mathrm{H}) .{ }^{13} \mathrm{C} \mathrm{NMR}: \delta-4.6(\mathrm{q})$, 18.4 (s), 26.5 (q), 31.6 (q), 55.1 (s), 55.8 (q), 56.1 (q), 60.2 (q), 100.00 (s), 102.1 (d), 104.3 (d), 109.2 (d), 118.7 (s), 120.1 (s), 123.3 (d), 125.5 (d), 126.1 (d), 126.2 (d), 128.8 (s), 129.5 (s), 131.4 (s), 133.2 (s), 147.1 (s), 147.5 (s), 149.5 (s), 150.9 (s). HRMS calculated for $\mathrm{C}_{31} \mathrm{H}_{41} \mathrm{NO}_{4} \mathrm{Si}$ : 519.2805. Found: 519.2800. NMR experiments (NOESY, HMBC and HMQC) confirmed the structure of the compound.<smiles>COc1ccc(OC)c2c(O)c3c(OC)c(NC(C)(C)C)c4ccccc4c3cc12</smiles>

8c: Yield: 98\%. ${ }^{1} \mathrm{H}$ NMR: $\delta 1.29$ (s, 9H), 2.97 (bs, 1H), 3.95 (s, 3H), 4.04 (s, 3H), 4.07 (s, 3H), 6.65 (m, 2H), 7.56 (m, 2H), 8.33 (m, 1H), 8.77 (m, 1H), 9.05 (s, 1H), 11.04 (s, 1H). ${ }^{13} \mathrm{C}$ NMR: $\delta 31.3$ (q), 56.1 (s), 56.2 (q), 57.1 (q), 61.6 (q), 102.2 (d), 103.3 (d), 107.1 (d), 113.8 (s), 113.9 (s), 124.0 (d), 125.8 (d), 126.0 (d), 126.3 (s), 127.0 (d), 129.3 (s), 130.5 (s), 131.3 (s), 133.6 (s), 148.4 (s), 150.6 (s), 150.8 (s), 151.3 (s). Anal. Calcd for $\mathrm{C}_{25} \mathrm{H}_{27} \mathrm{NO}_{4}$ : C, 74.05; H, 6.71; N, 3.45. Found: C, 74.25; H, 6.69; N, 3.49. HRMS calculated for $\mathrm{C}_{25} \mathrm{H}_{27} \mathrm{NO}_{4}$ : 405.1940. Found: 405.1935 .

\section{References}

1.- Lautens, M.; Schmid, G.A.; Chau, A. J. Org. Chem. 2002, 67, 8043-8053.

2.- (a) Dötz, K.H.; Kuhn, W.J. J. Organomet. Chem. 1985, 286, C23-C26. (b) Chen, K.S.; Wulff, W.D. J. Am. Chem. Soc. 1986, 108, 5229-5236. (c) Barluenga J.; Aznar, F.; Barluenga, S.; Fernández, M.; Martín, A.; García-Granda, S.; Piñera-Nicolás, A. Chem. Eur. J. 1998, 4, 2280-2298. (d) Barluenga, J.; Aznar, F.; Palomero, M.A. Chem. Eur. J. 2002, 8, 4149-4163. 
${ }^{1 \mathrm{H}}$ and ${ }^{13} \mathrm{C}$ NMR spectra of selected compounds $\left(\mathrm{CDCl}_{3}\right)$.

芲

ณ̊ 응

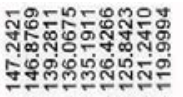

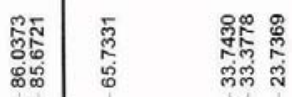<smiles>COC(=C1C(C2=CCCC2)=C(C2=CCCC2)C2OC1c1ccccc12)C(Cl)(Cl)Cl</smiles>

$4 a$
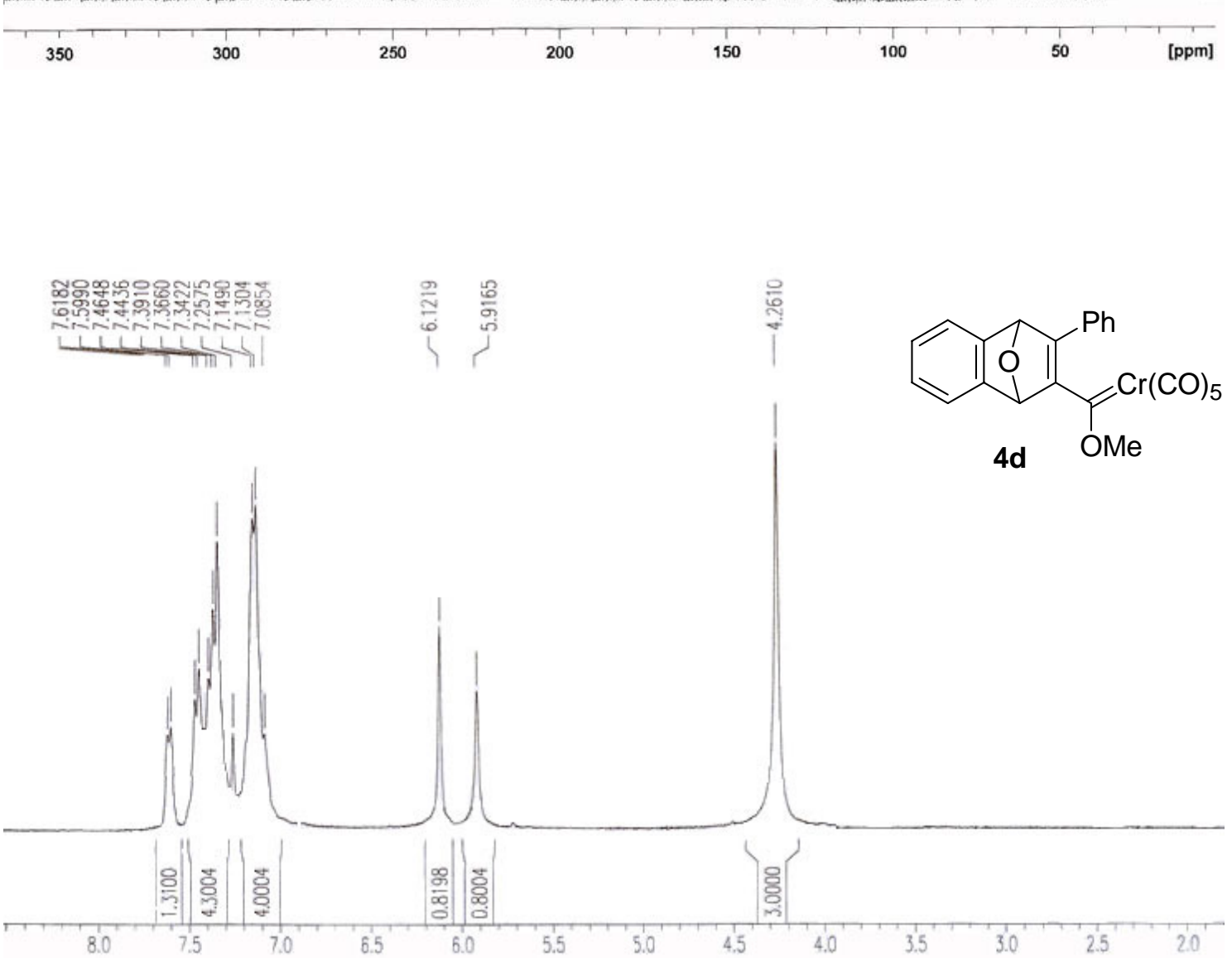


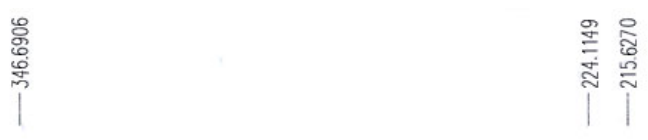

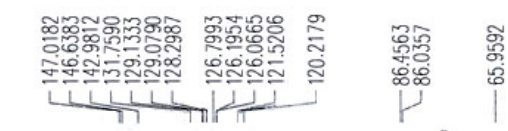<smiles>COC(=C1C2OC(c3ccccc32)C1c1ccccc1)C(Cl)(Cl)Cl</smiles>

4d

Me

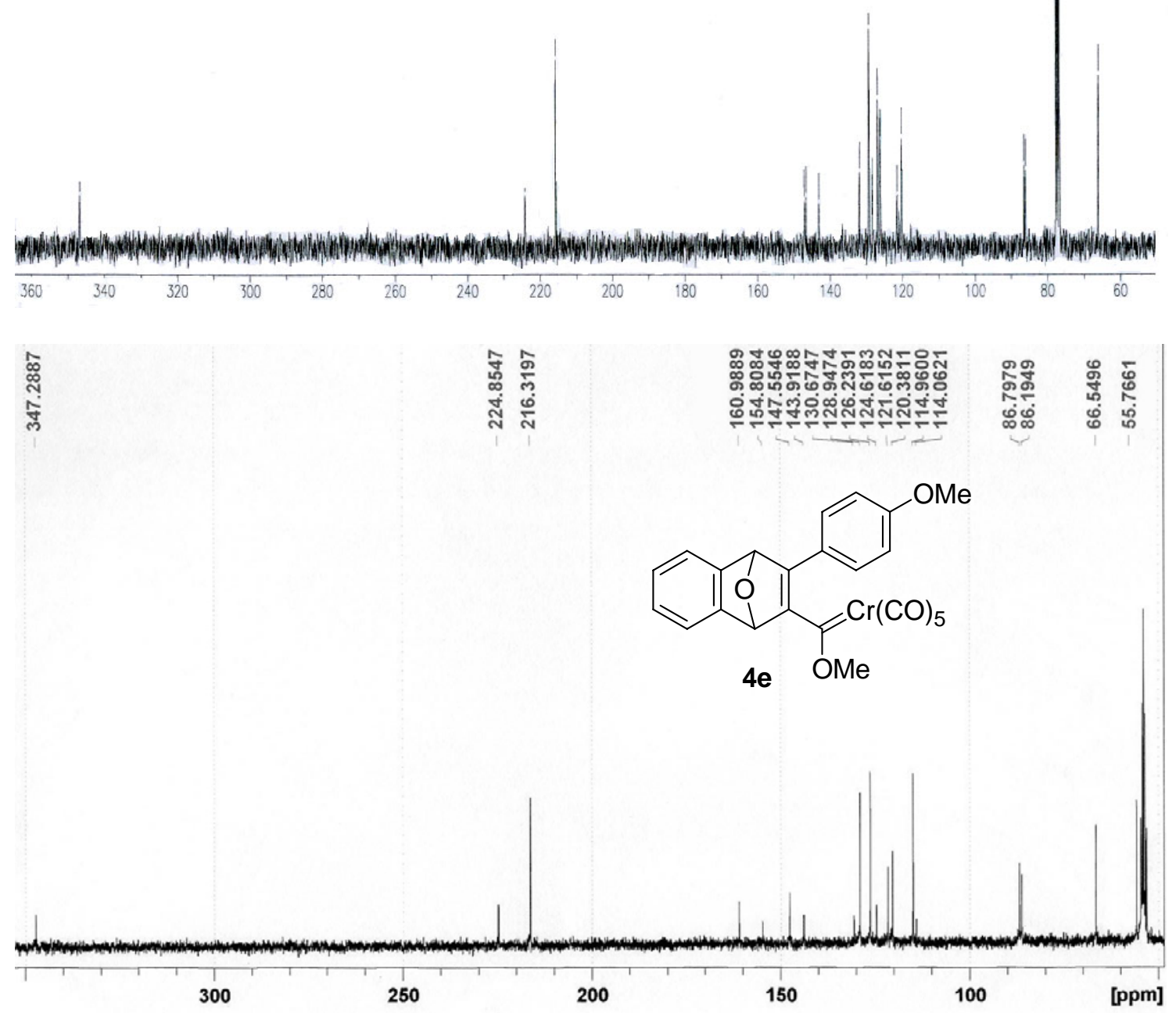



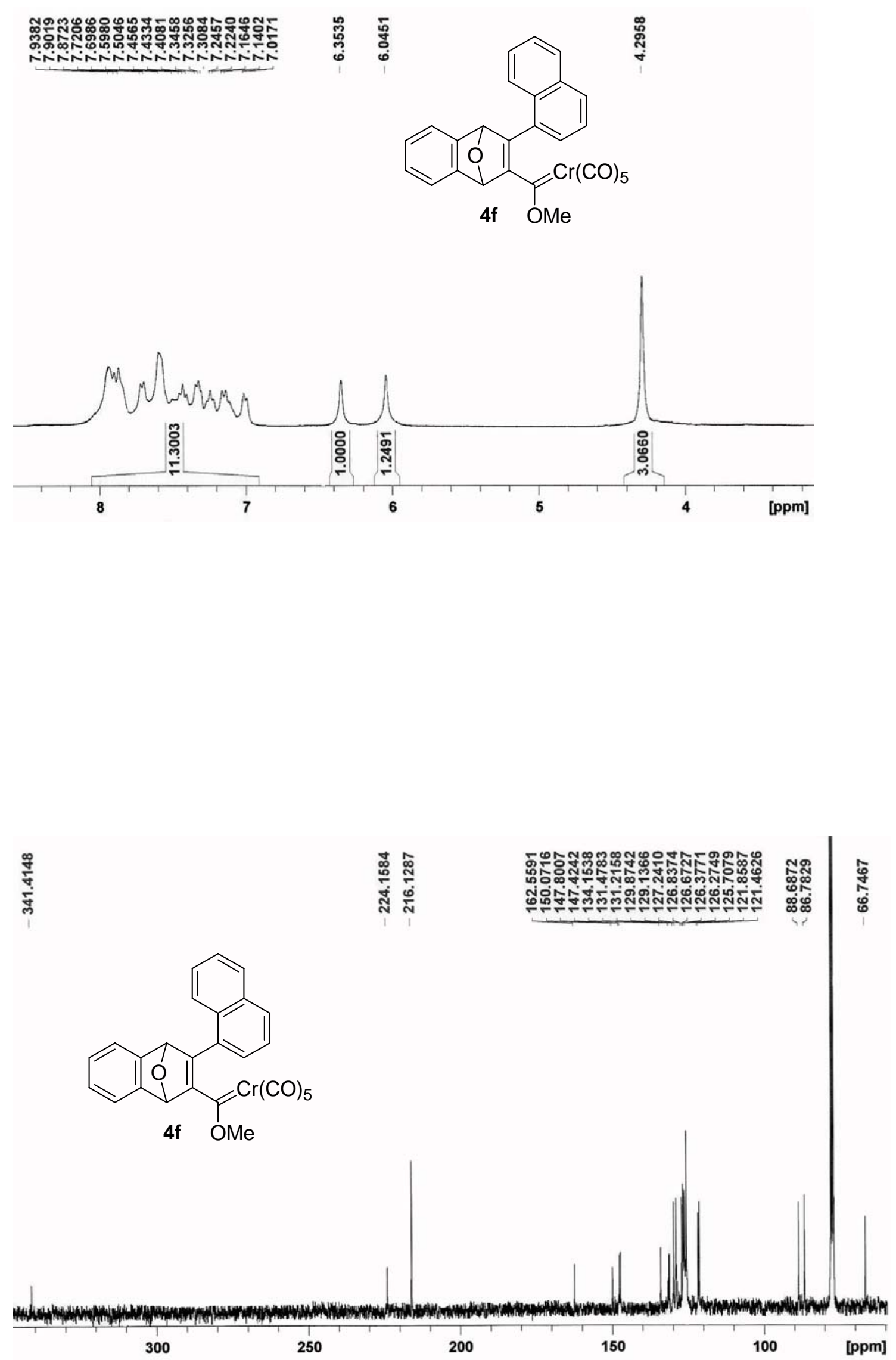


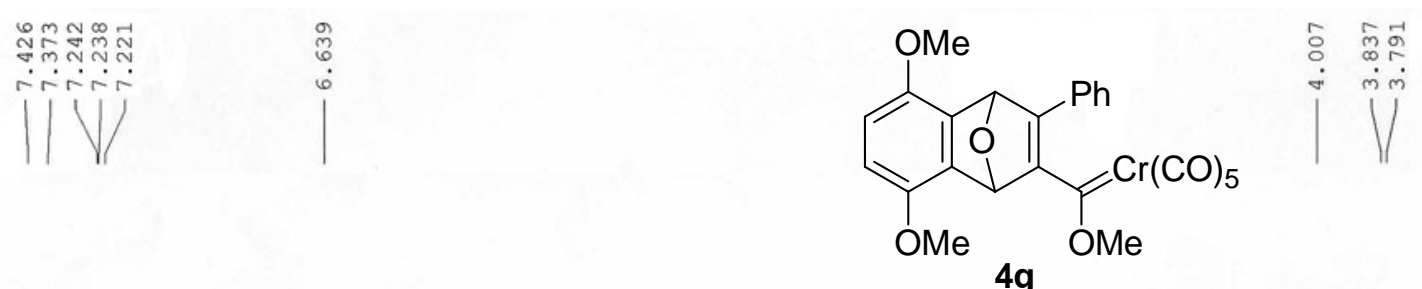

$4 \mathrm{~g}$
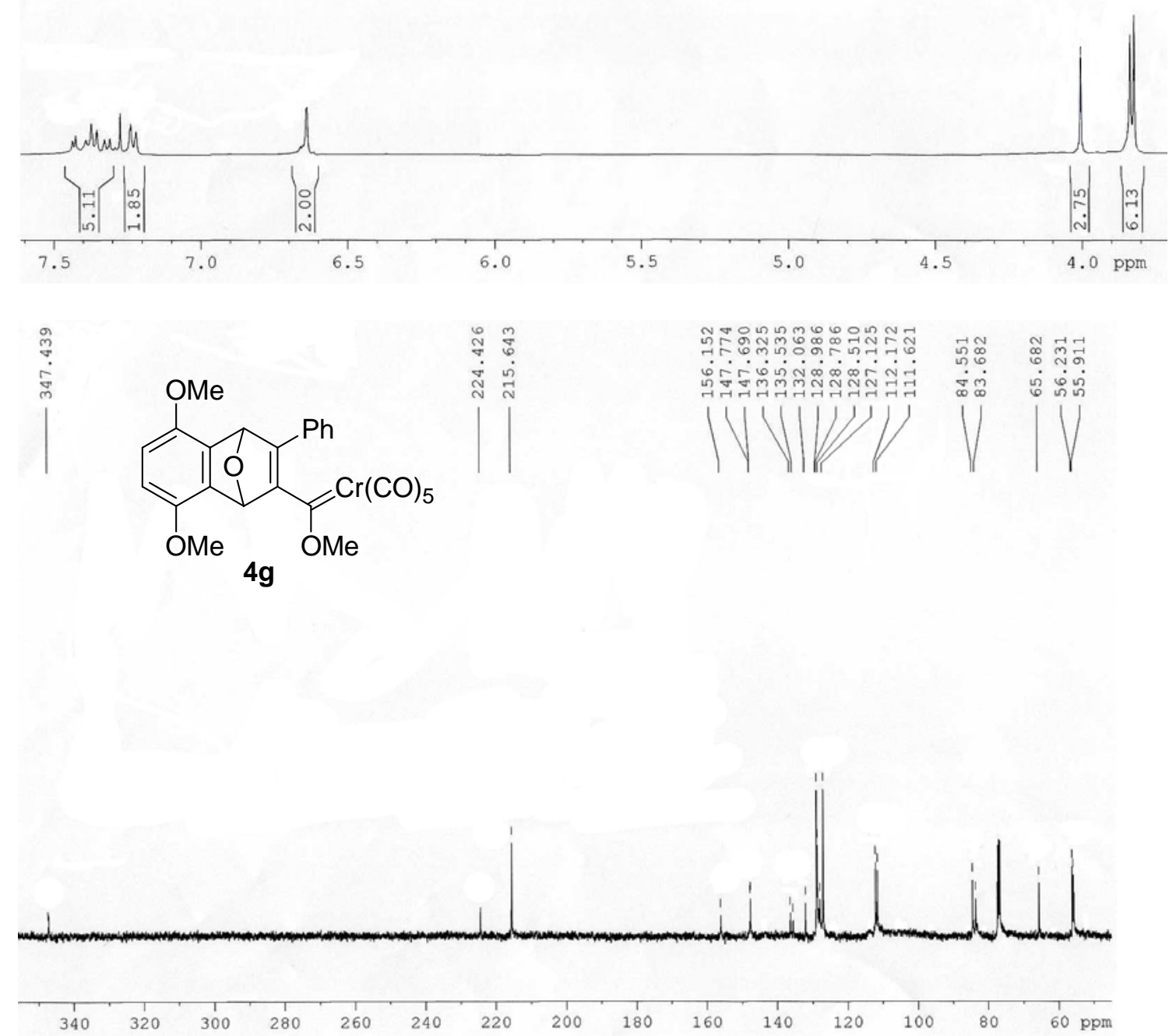
<smiles>COC1=C(C(=C(C(=O)[O-])C(C)(F)F)c2ccc(Cl)cc2)C2OC1c1ccccc12</smiles>

$4 h$

OMe

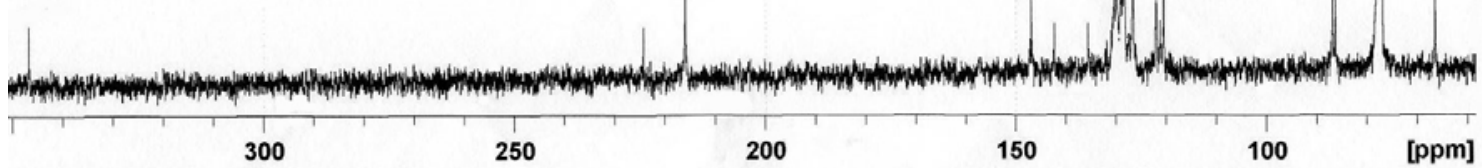

旁

능 ํํํ

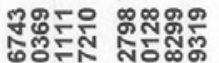

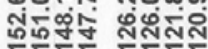

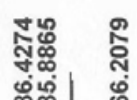

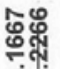

난

$\infty \infty$<smiles>COC(=O)C(C(=O)[O-])=C(OC)C1=C(C(C)(C)C)C2OC1c1ccccc12</smiles>

$4 i$

OMe

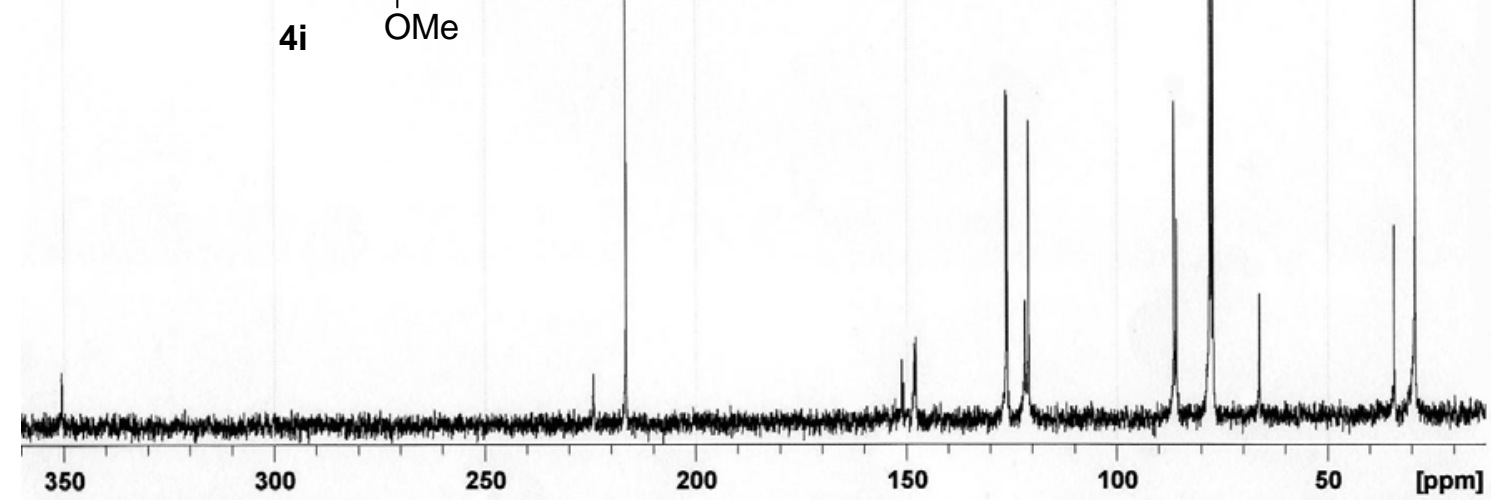




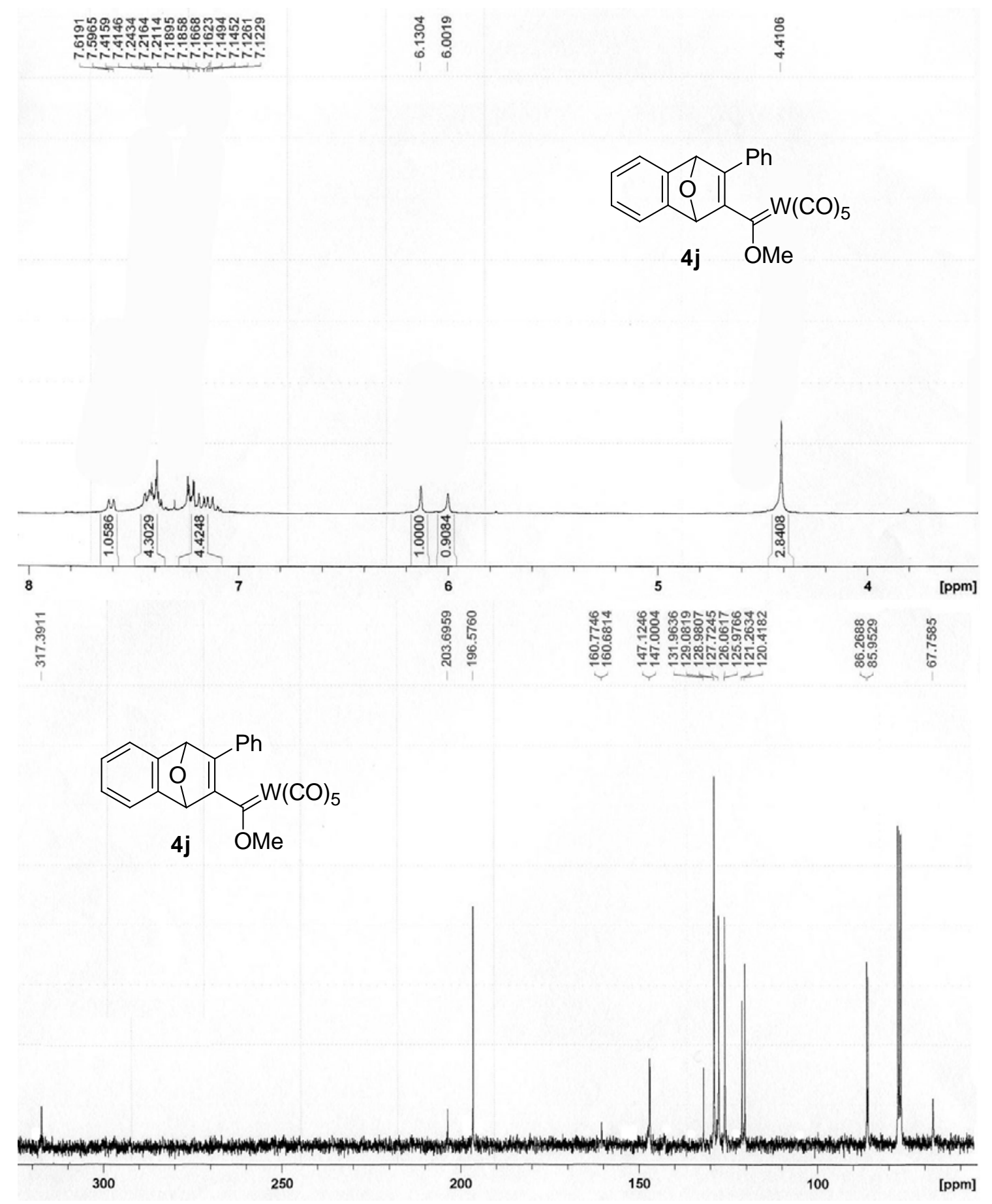



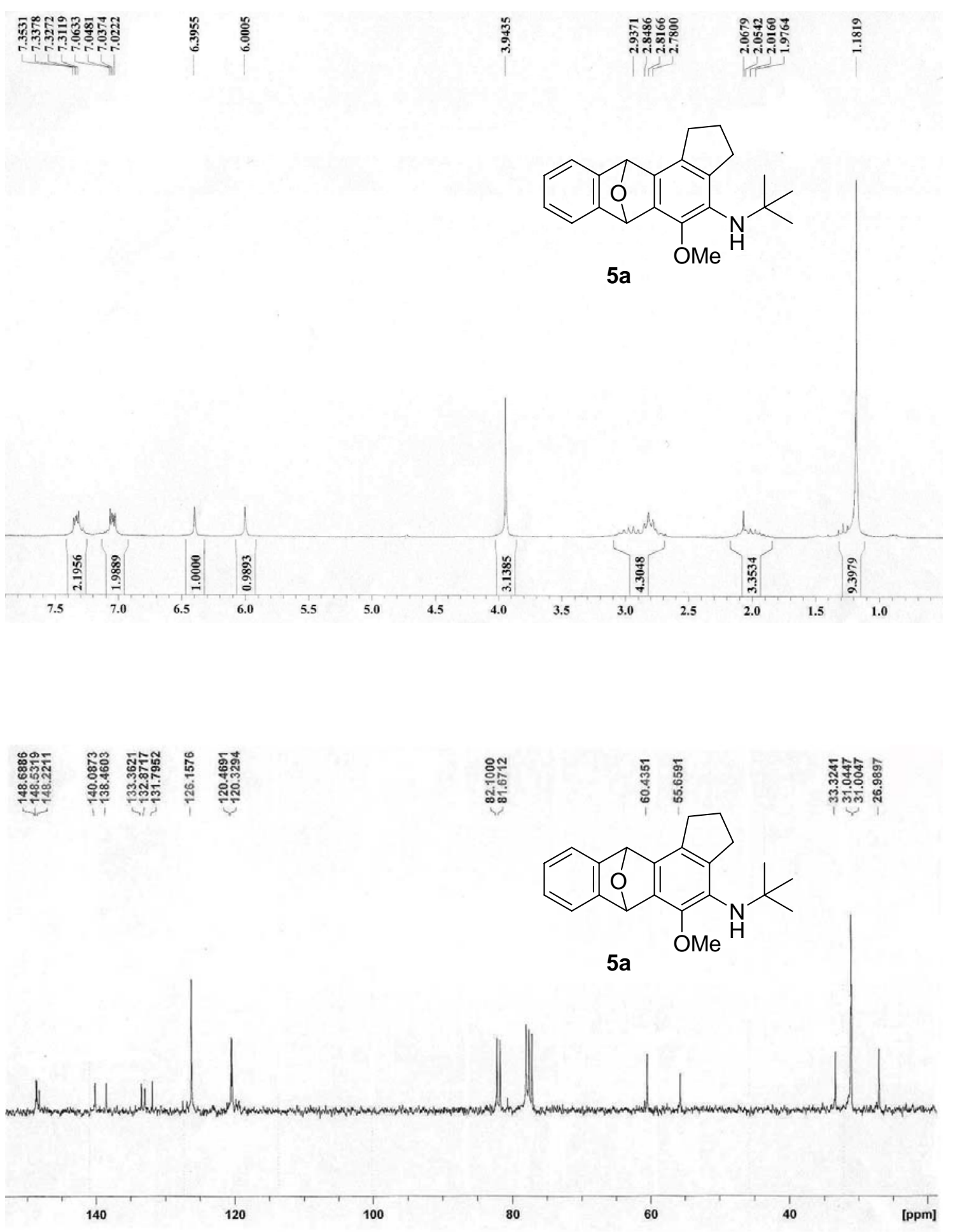


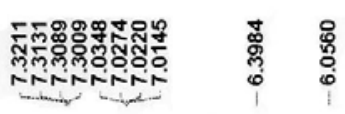

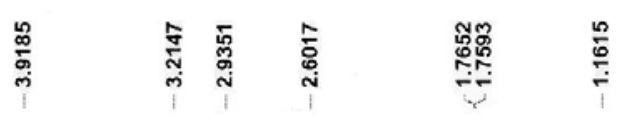<smiles>COc1c(NC(C)(C)C)c2c(c3c1C1OC3c3ccccc31)CCCC2</smiles>

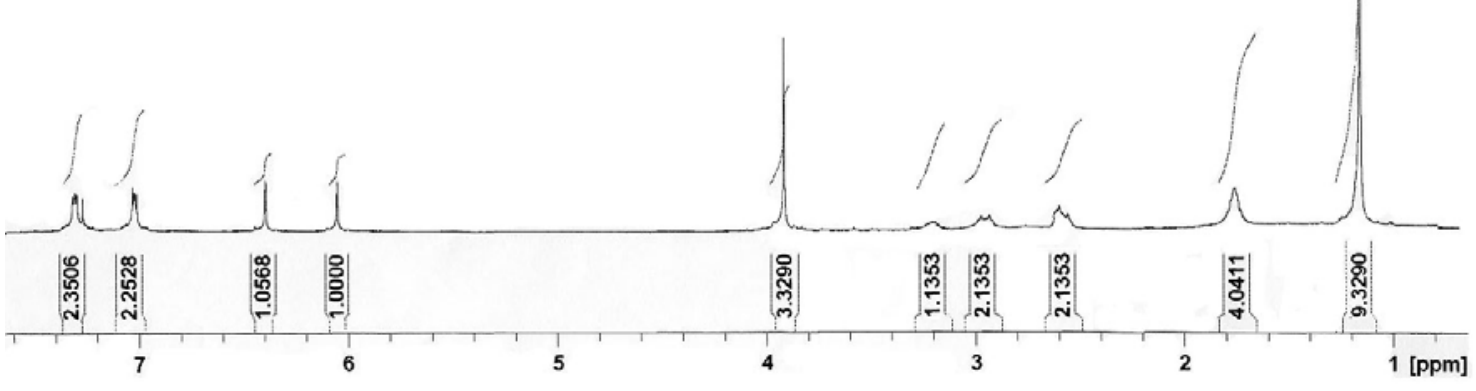

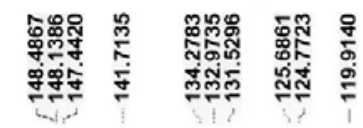

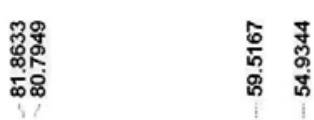

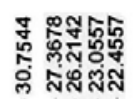

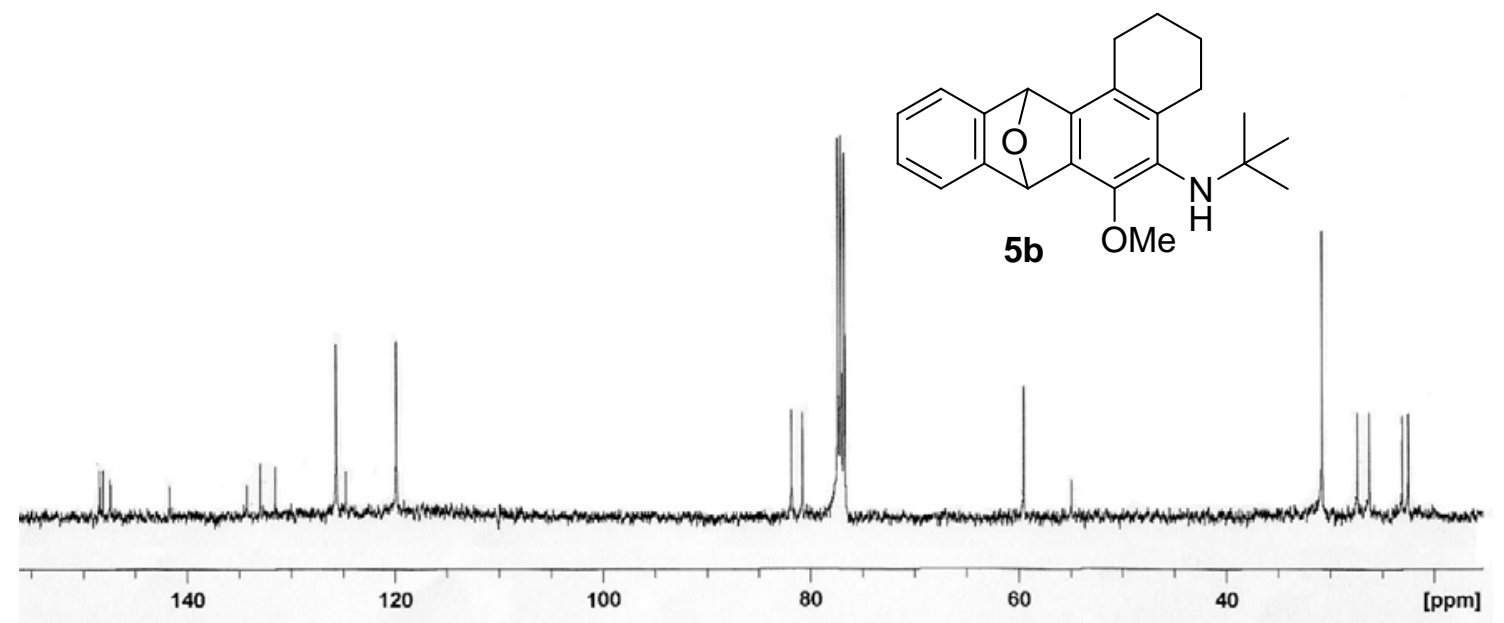



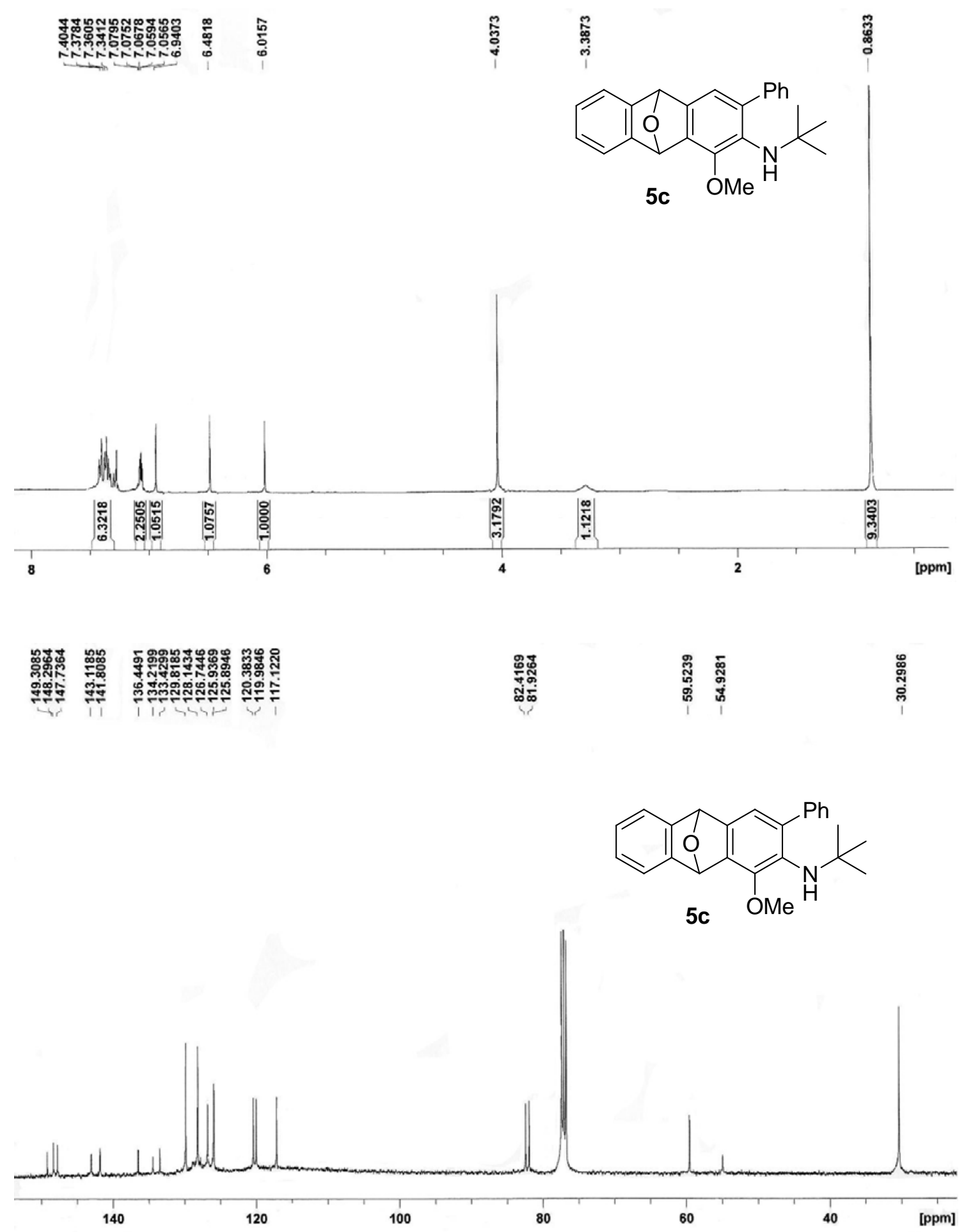


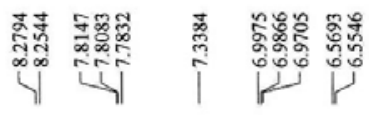

旁旁<smiles>COc1c2c(c3ccccc3c1NC(C)(C)C)C1OC2c2ccccc21</smiles>
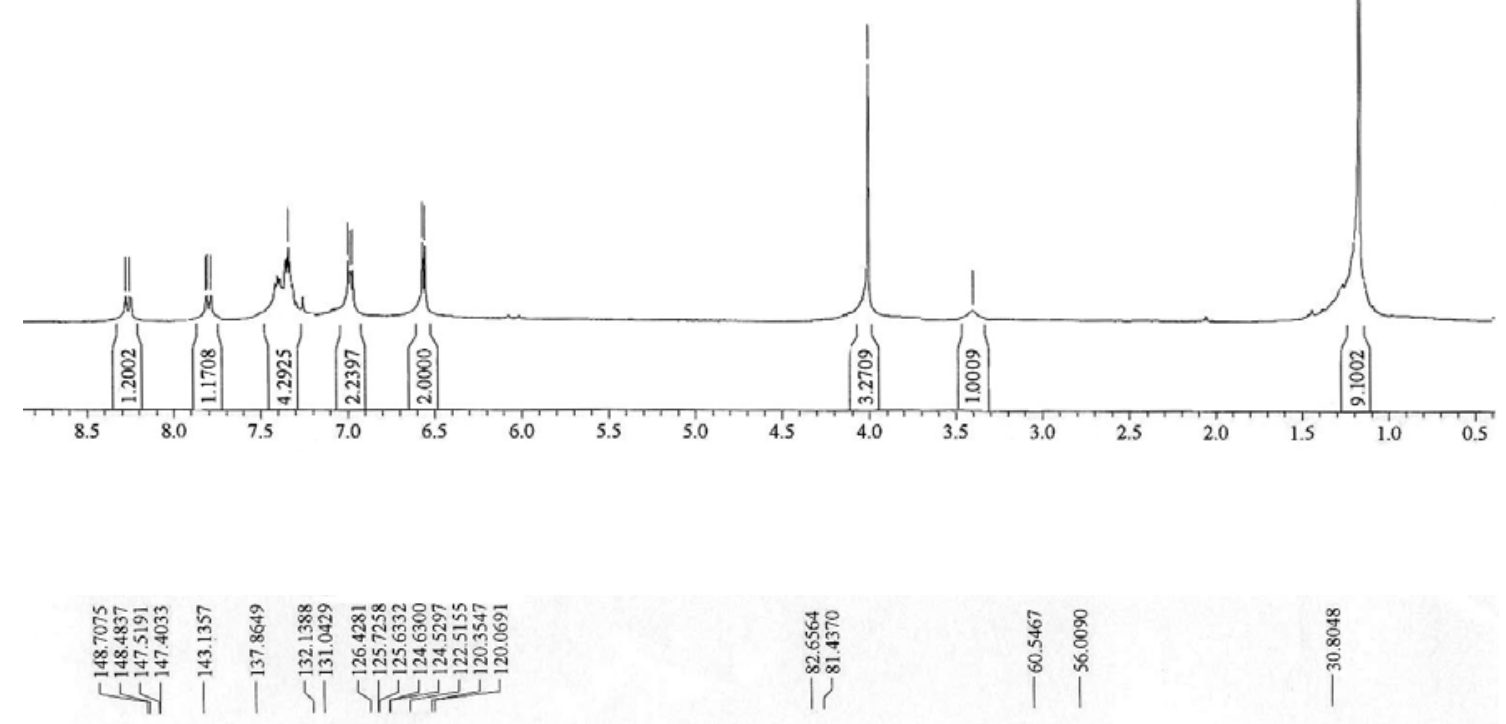

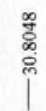

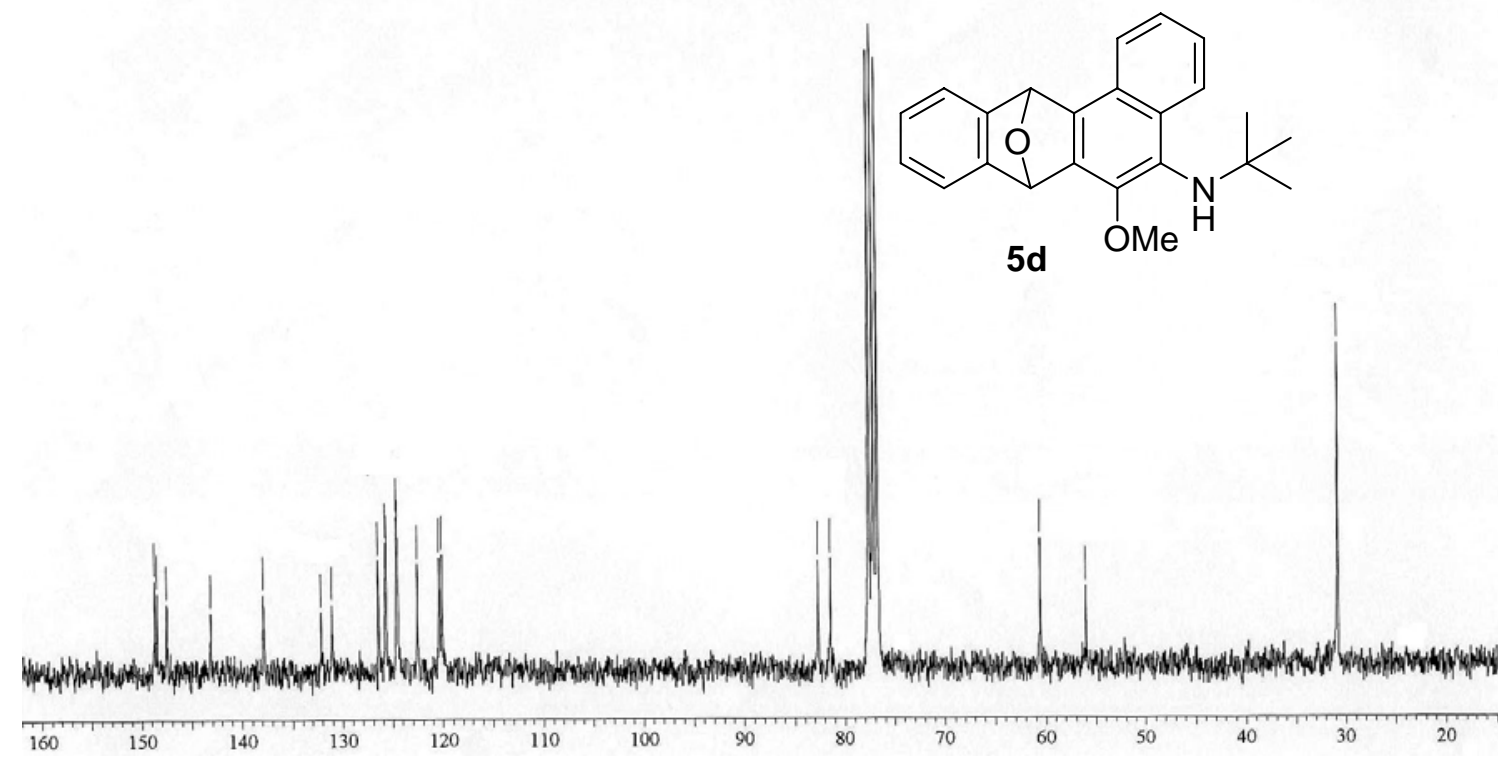




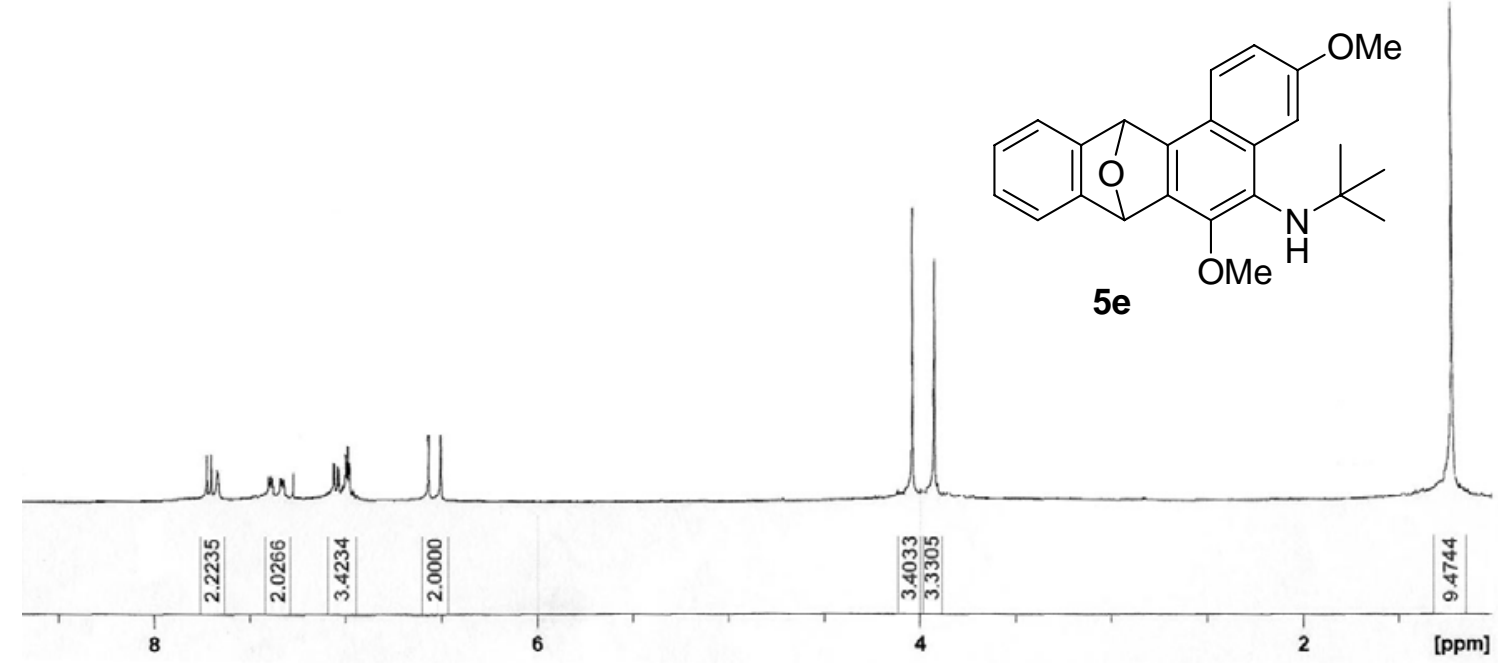<smiles>COc1ccc2c3c(c(OC)c(NC(C)(C)C)c2c1)C1OC3c2ccccc21</smiles>
$5 e$

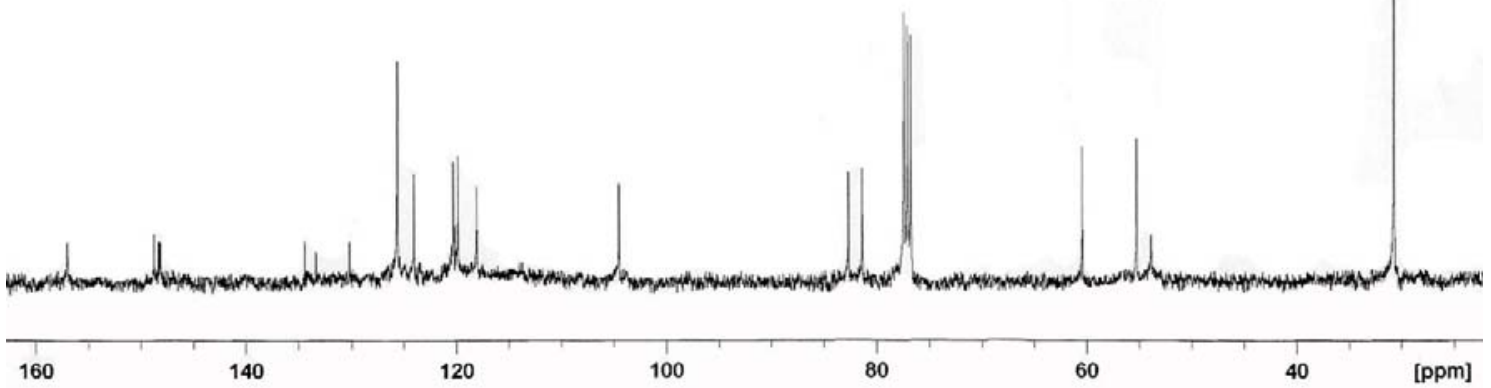



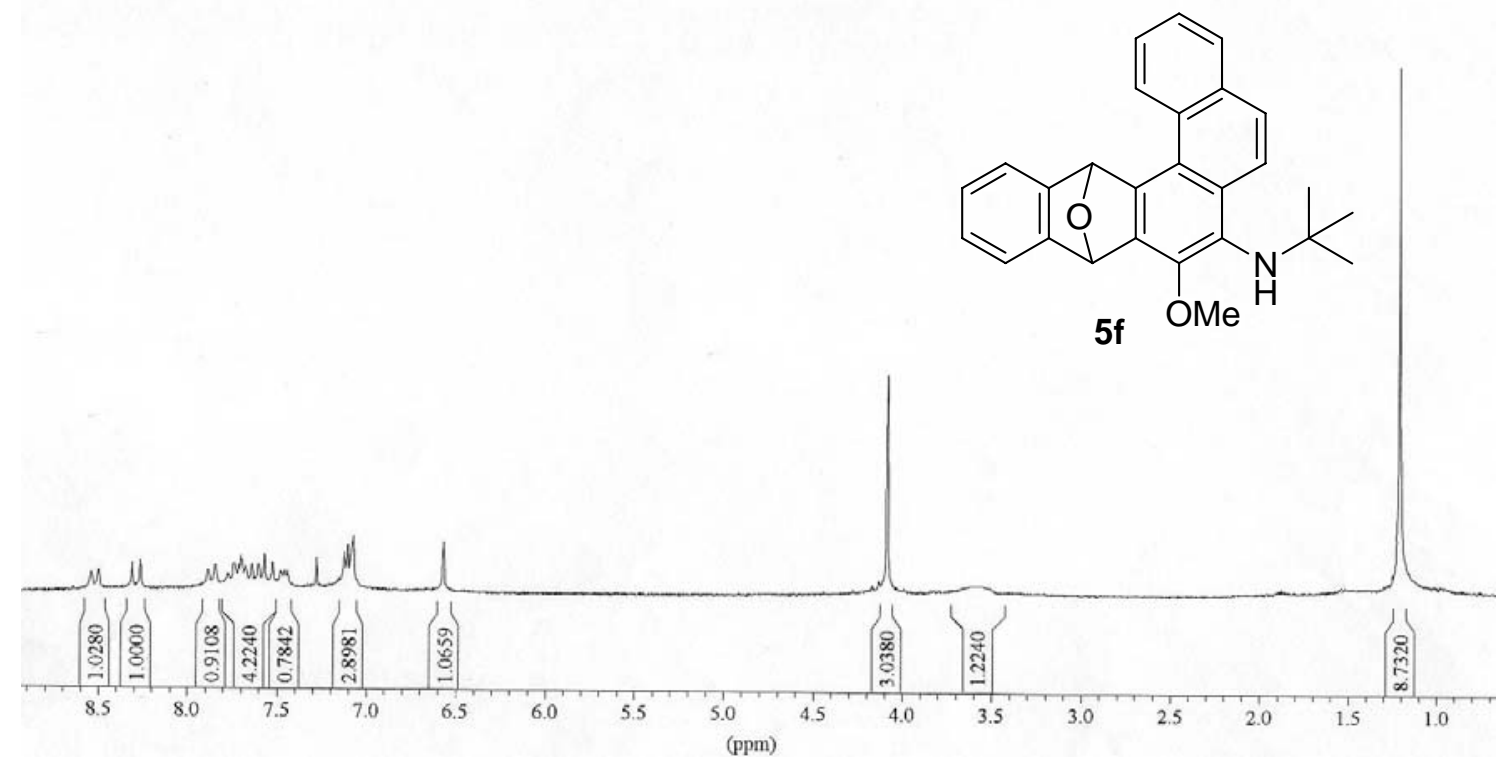

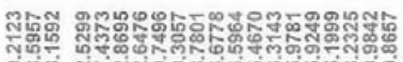

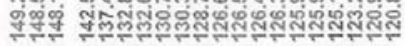

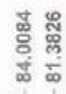

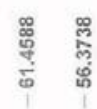

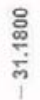<smiles>COc1c2c(c3c(ccc4ccccc43)c1NC(C)(C)C)C1CC2OC1Br</smiles>

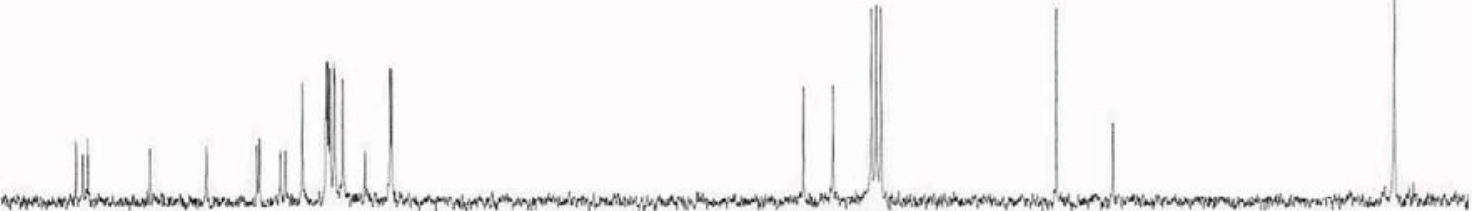

140

120

100

80

60

[ppm] 

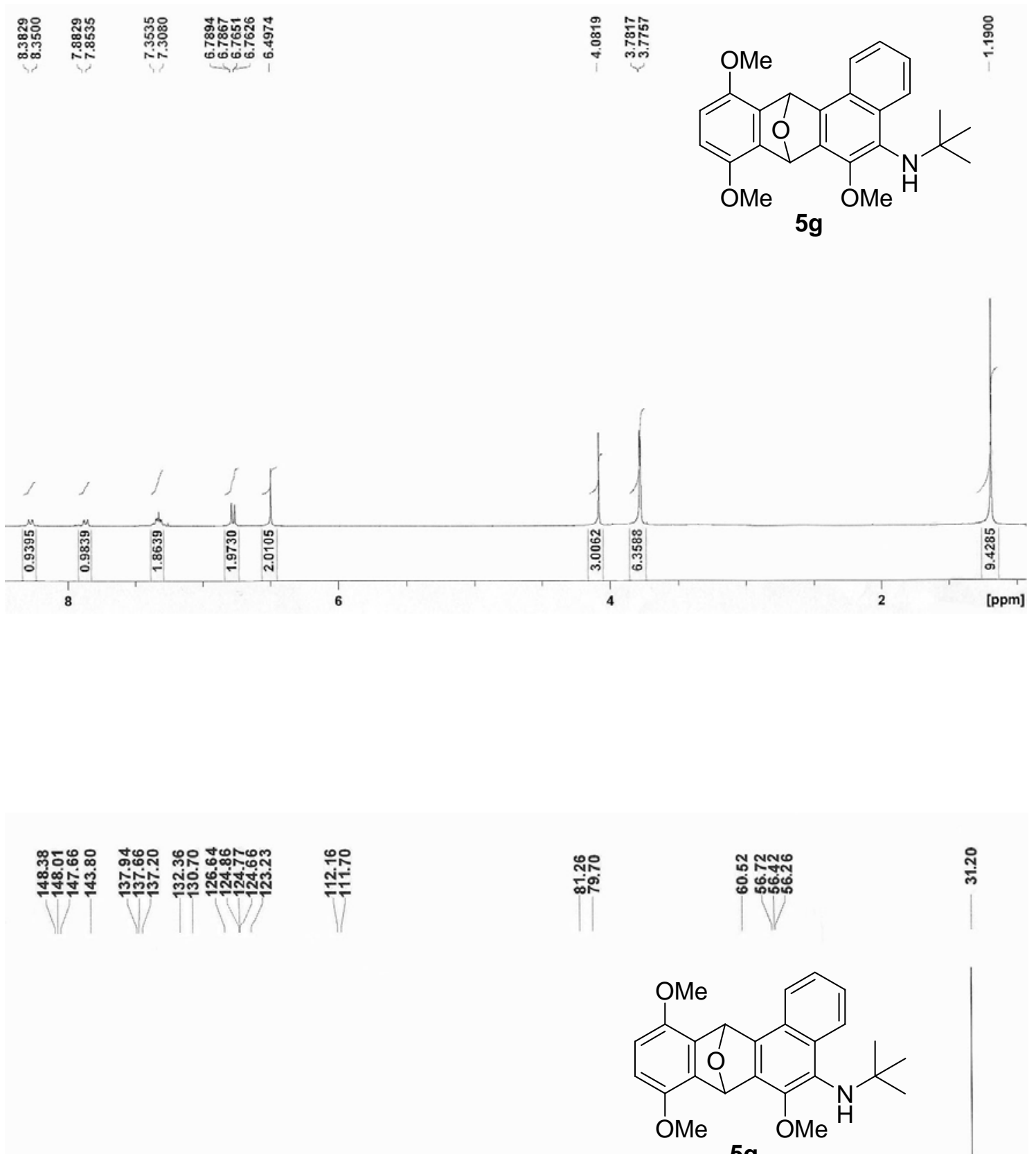

$5 g$
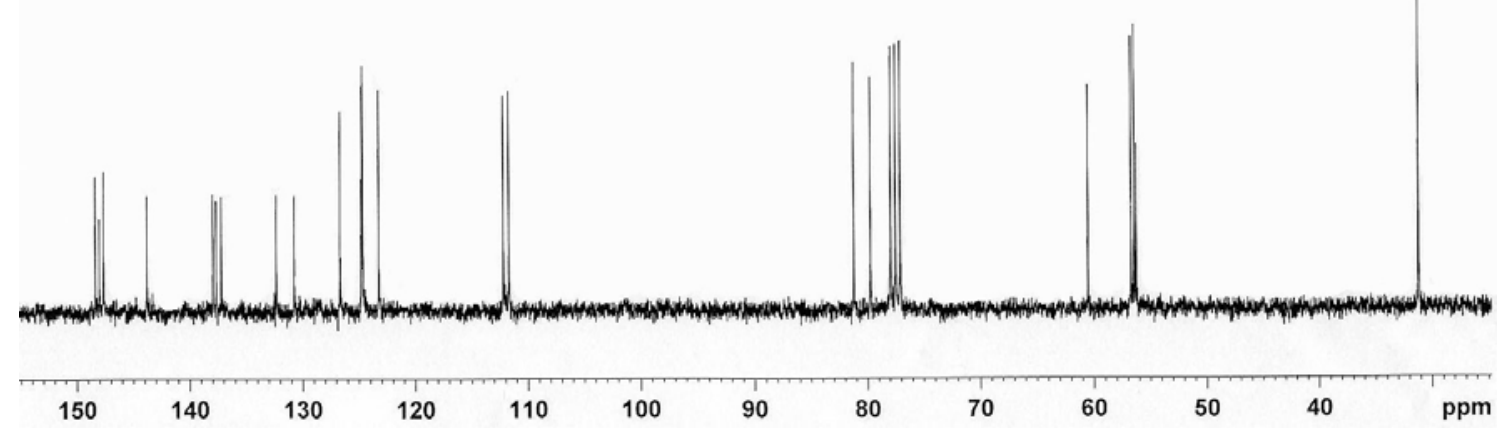


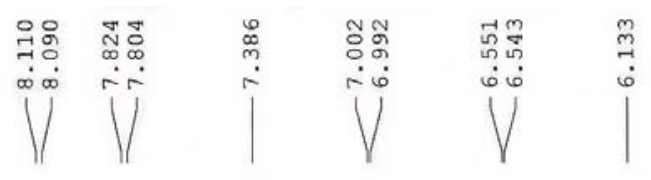<smiles>COc1c2c(c3ccccc3c1O)C1OC2c2ccccc21</smiles>
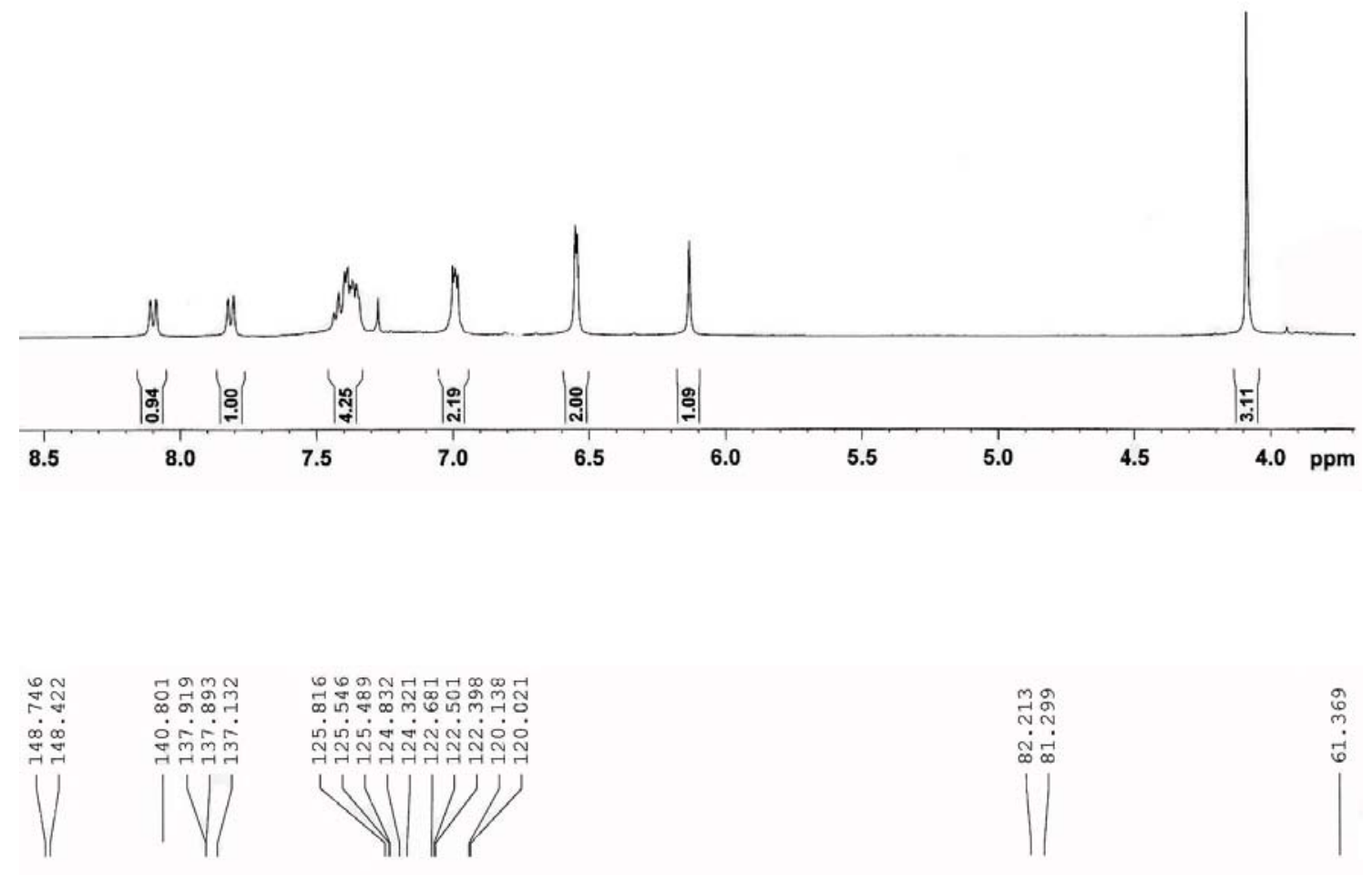<smiles>COc1c2c(c3ccccc3c1O)C1OC2c2ccccc21</smiles>

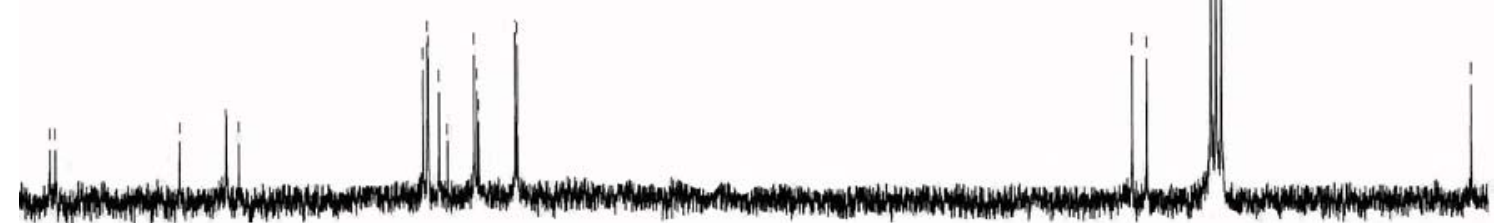

$\begin{array}{llllllllllllllllll}145 & 140 & 135 & 130 & 125 & 120 & 115 & 110 & 105 & 100 & 95 & 90 & 85 & 80 & 75 & 70 & 65 & \mathrm{ppm}\end{array}$ 


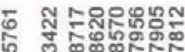

?
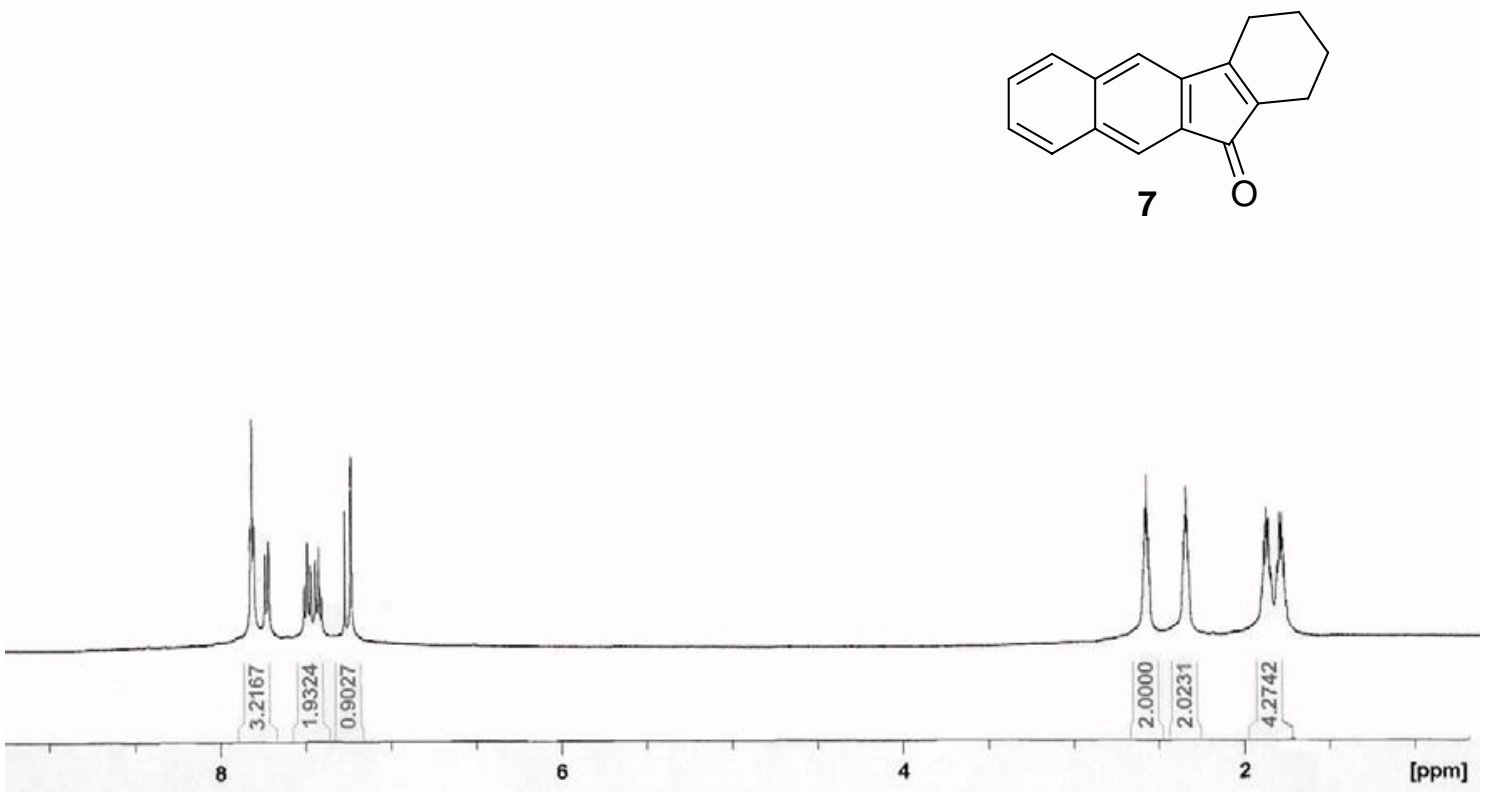

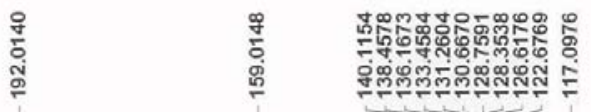
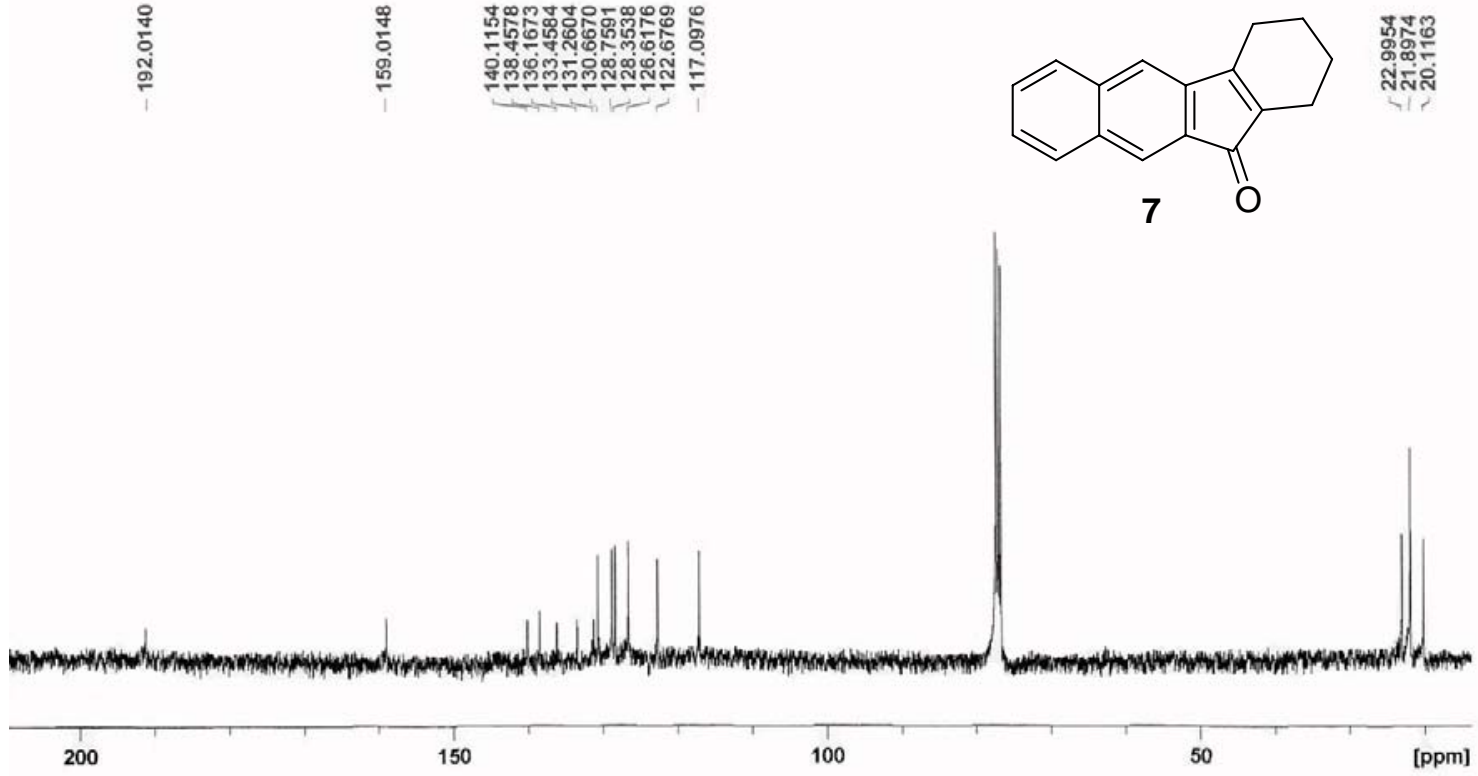


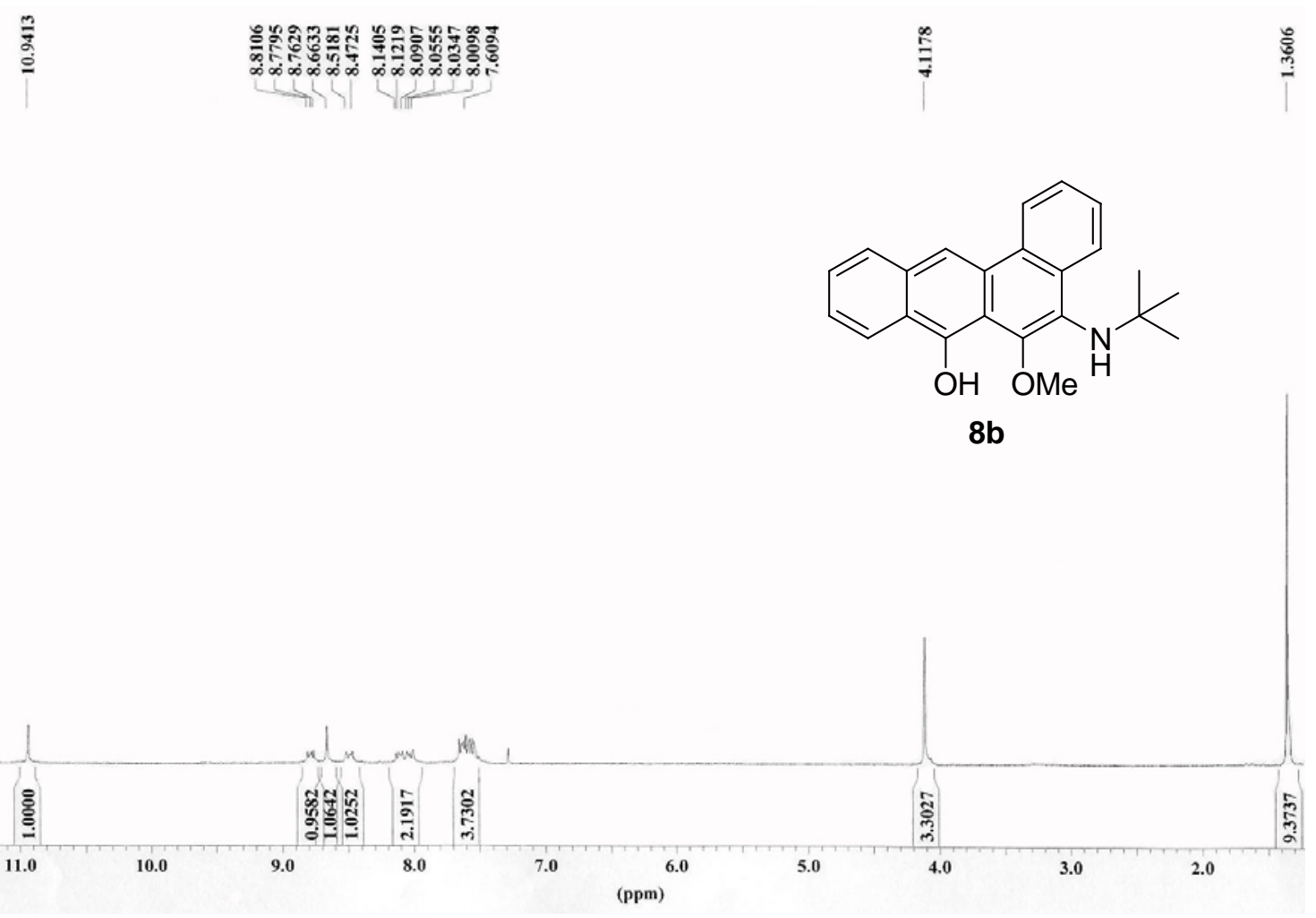

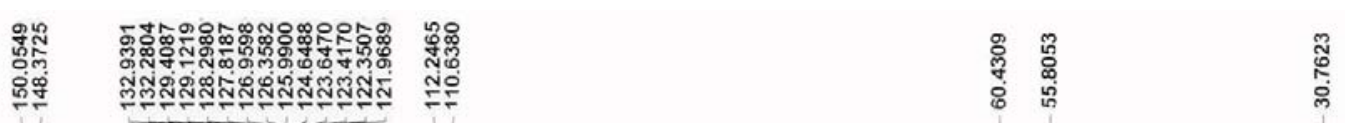

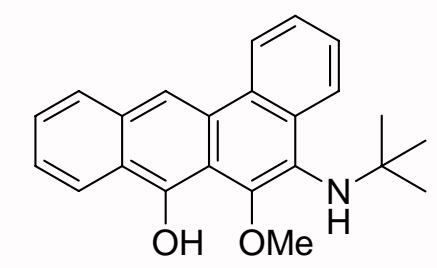

$8 b$

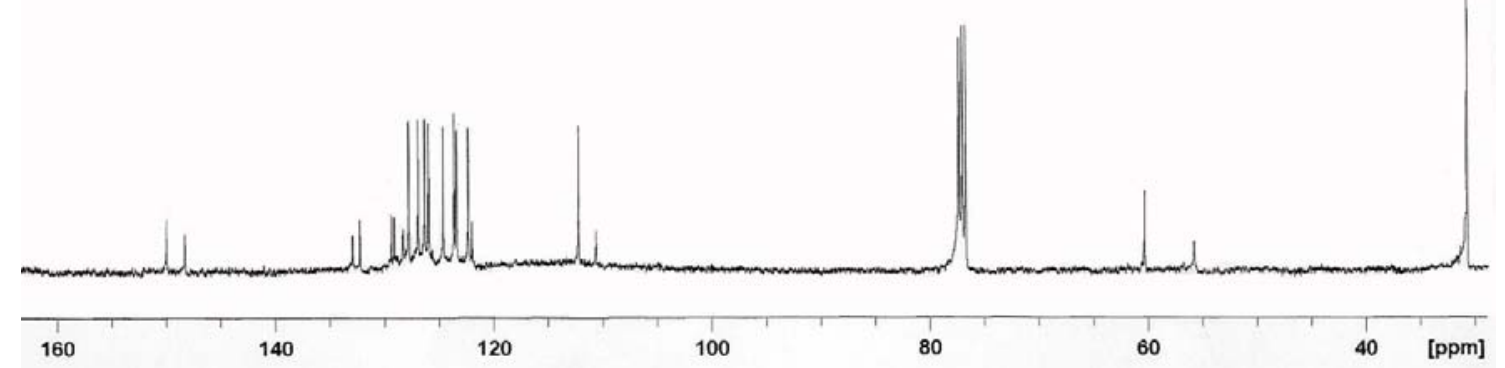




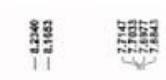

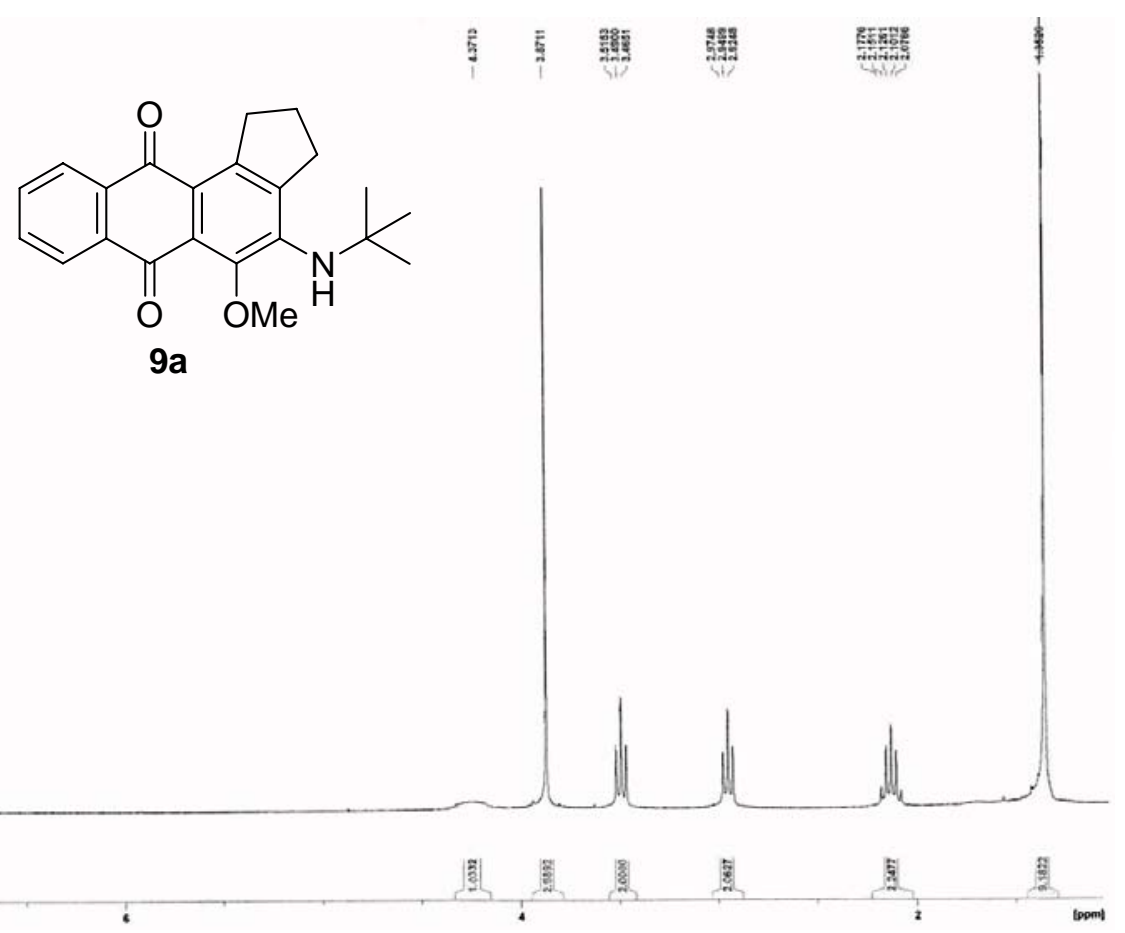

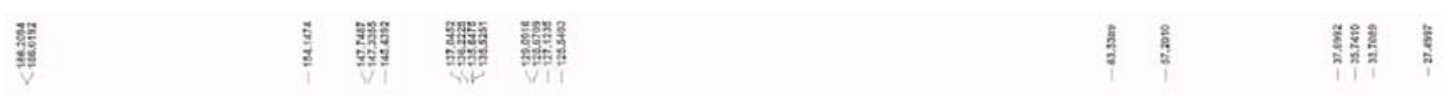<smiles>COc1c(NC(C)(C)C)c2c(c3c1C(=O)c1ccccc1C3=O)CCC2</smiles>

$9 a$

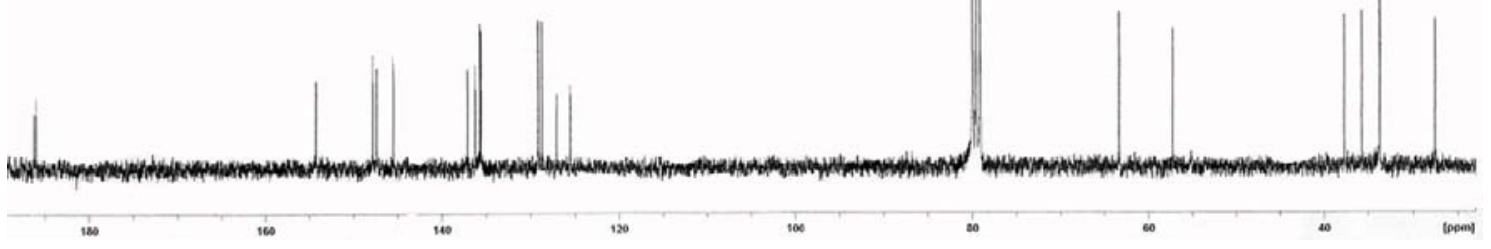



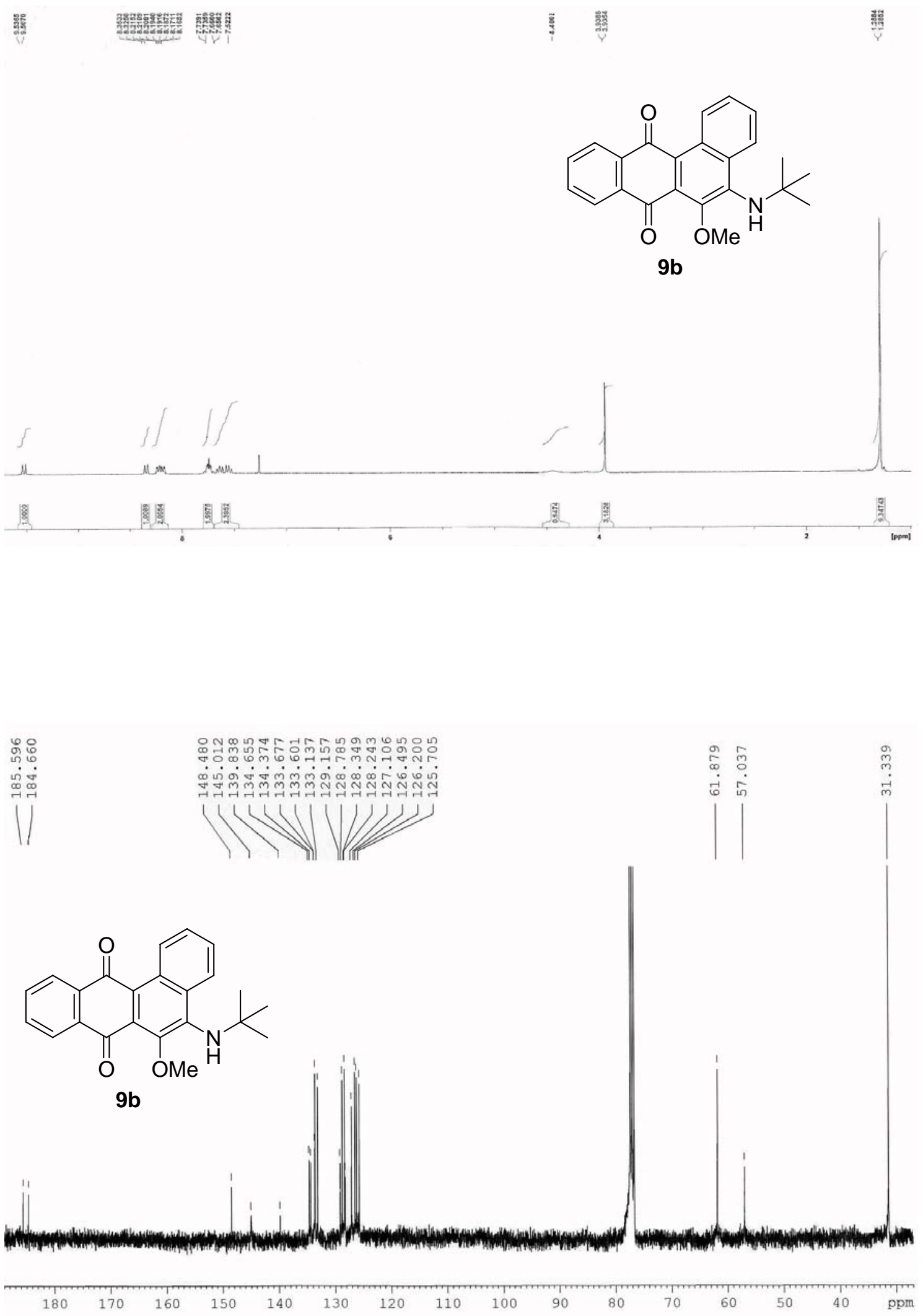
<smiles>COc1ccc(OC)c2c(O)c3c(OC)c(NC(C)(C)C)c4ccccc4c3cc12</smiles>

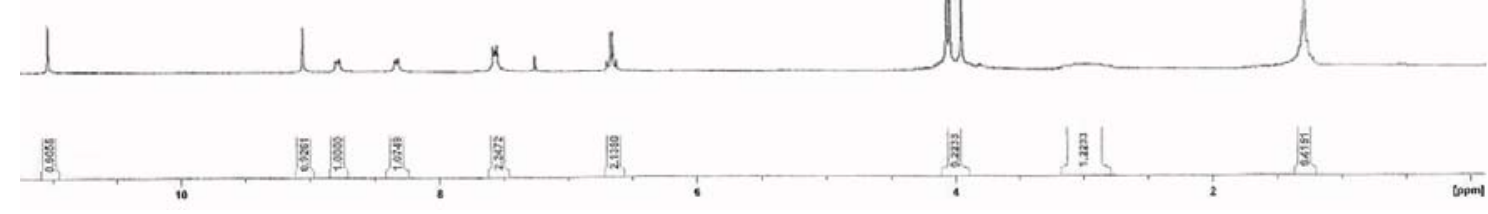<smiles>COc1ccc(OC)c2c(O)c3c(OC)c(NC(C)(C)C)c4ccccc4c3cc12</smiles>
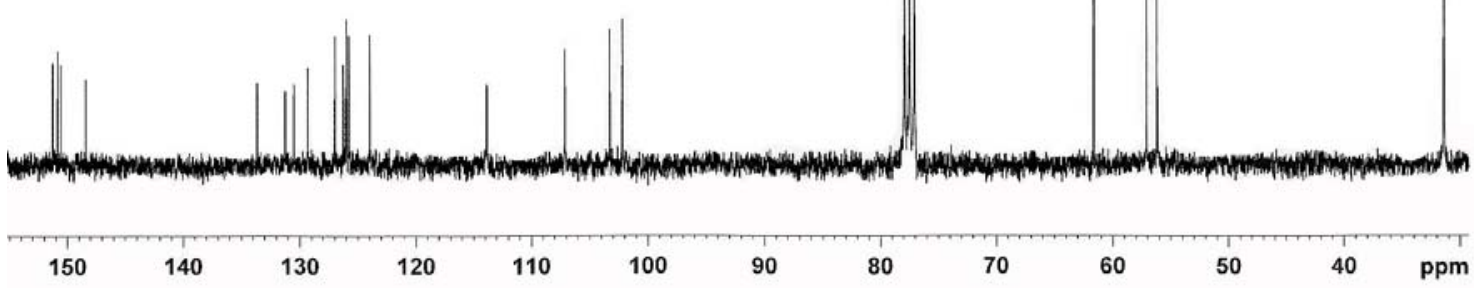

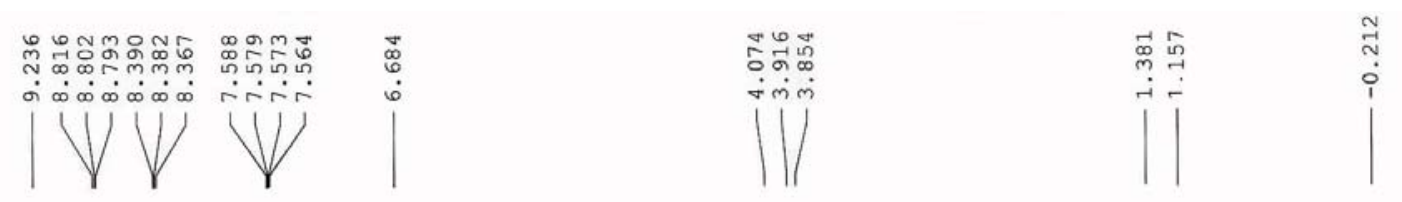<smiles>COc1ccc(OC)c2c(OC)c3c(OC)c(NC(C)(C)C)c4ccccc4c3cc12</smiles>

O-Silyl-8c
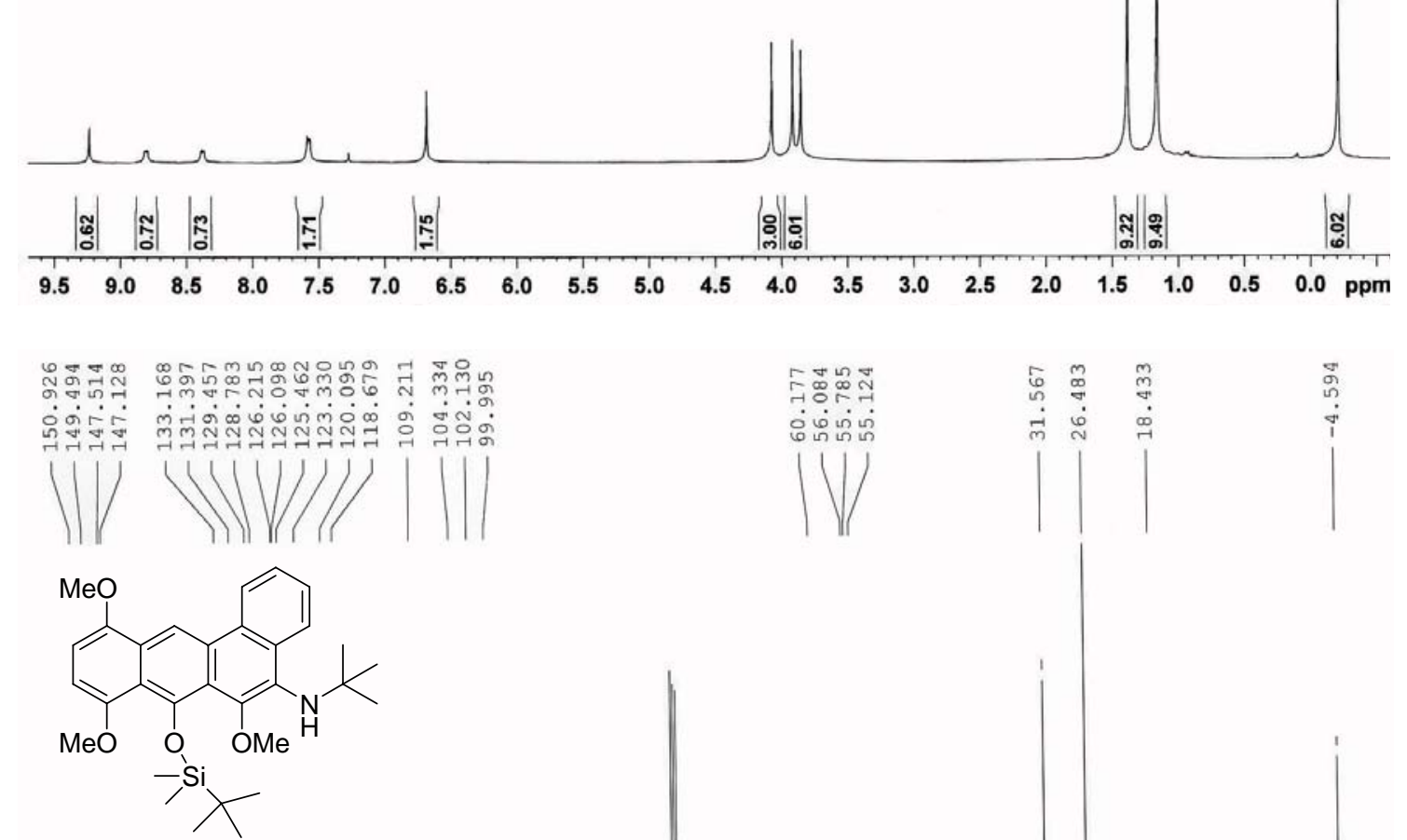

O-Silyl-8c

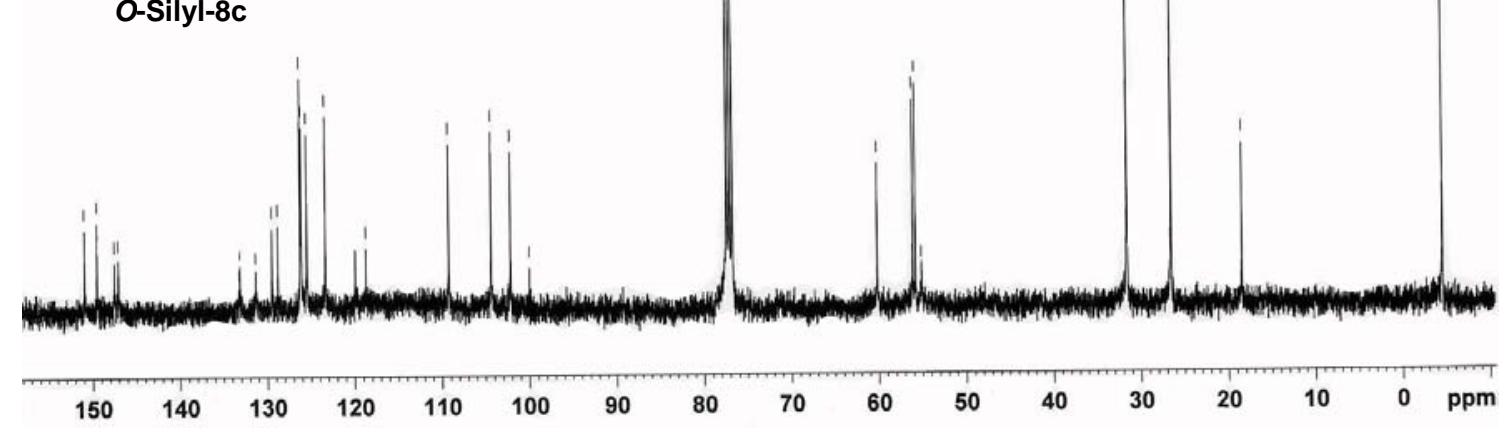

